\title{
30. PETROLOGY AND GEOCHEMISTRY OF ARC THOLEITTES ON THE PALAU-KYUSHU RIDGE, SITE 448, DEEP SEA DRILLING PROJECT LEG 59
}

\author{
Robert B. Scott, Department of Geology, Texas A\&M University, College Station, Texas
}

\section{INTRODUCTION}

This chemical and petrologic study of rocks from Site 448 on the Palau-Kyushu Ridge is designed to answer some fundamental questions concerning the volcanic origin of remnant island arcs. According to the reconstruction of the Western Pacific prior to about 45 m.y. ago (Hilde et al., 1977), the site of the Palau-Kyushu Ridge was a major transform fault. From a synthesis of existing geological and geophysical data (R. Scott et al., this volume), it appears that the ridge originated by subduction of the Pacific plate under the West Philippine Basin. Thus the Palau-Kyushu Ridge should be a prime example of both initial volcanism of an incipient arc formed by interaction of oceanic lithospheric plates and remnant-arc volcanic evolution. The Palau-Kyushu Ridge was an active island arc from about 42 to $30 \mathrm{~m}$.y. ago, after which initiation of back-arc spreading formed the Parece Vela Basin (R. Scott et al., this volume; Karig, 1975a). This spreading left the western portion of the ridge as a remnant arc that separates the West Philippine Basin from the Parece Vela Basin. In spite of numerous oceanographic expeditions to the Philippine Sea, including the two previous DSDP Legs 6 and 31 (Fischer, Heezen et al., 1971; Karig, Ingle et al., 1975), and even though the origins of inter-arc basins have been linked by various hypotheses to that of remnant island arcs (Karig, 1971, 1972, 1975a, and 1975b; Gill, 1976; Uyeda and Ben-Avraham, 1972; Hilde et al., 1977), very little hard data are available on inactive remnant arcs.

Continental orogenic belts contain complexes such as those in Newfoundland and the Caucasus Mountains that have been interpreted as structural fragments of marginal basin-magmatic arc complexes. Thus recognition of processes involved in continental-crust generation requires a thorough understanding of the genesis of both remnant arcs and marginal basins. To this end several questions must be addressed: (1) Is the PalauKyushu Ridge actually a remnant arc? (2) If so, does this remnant arc have any petrologic features unique to the related marginal-basin formation? (3) Does the arc represent the initial phases of magmatic-arc evolution; that is, does volcanism on the Palau-Kyushu Ridge have an arc-tholeiitic character followed by progressively more calc-alkalic volcanism on the West Philippine and Mariana Ridges? Or is the initial arc-tholeiite-magmatic phase repeated each time a new arc is formed after sundering of the previous arc? (4) Is it possible to use the combination of petrologic and geomorphologic characteristics of the Palau-Kyushu Ridge to specify how and where the magmatic arc was sundered? (5) What is the temporal relationship between the cessation of arc volcanism and the sundering of the arc (with subsequent back-arc basin formation)? (6) Is there any indication that immature, largely submarine arcs have established widespread hydrothermal activity that may be the precursor of ore-deposition mechanisms characteristic of mature island arcs?

The most extensive petrologic studies of PalauKyushu Ridge rocks prior to Leg 59 were reported by the International Working Group on the IGCP Project "Ophiolites" (1977), which used suites of samples collected during cruise 17 of the Dmitry Mendeleev between June and August of 1976. Rocks were collected from two dredge stations (1397 and 1396) located on the steep western face of the Palau-Kyushu Ridge (see Site 448 report for a location map, this volume). The rocks collected included subalkalic basalts, basaltic andesites, andesites, two-pyroxene gabbros, and low-grade pumpellyite-prehnite or greenschist-facies hydrothermal metamorphic rocks. These volcanic rocks are reported to be highly vesicular, typical of shallow-marine or even subaerial volcanism. Two analyses of typical basaltic andesites are presented in Table 1 for comparison with our own findings. The International Working Group concluded that the highly vesicular nature and the petrologic trends of these rocks are typical of island-arc magmatic products. The high $\mathrm{Fe} / \mathrm{Mg}$ ratios and low $\mathrm{Ni}$, $\mathrm{Cr}, \mathrm{Sr}$, and $\mathrm{Ba}$ contents are suggestive of arc tholeiites. The high $\mathrm{K}$ is probably the result of sea-water weathering. The low $\mathrm{Ba}$ and $\mathrm{Sr}$ and high $\mathrm{FeO} / \mathrm{FeO}+\mathrm{MgO}$ ratio also rule out the possibility that these may be calcalkalic series differentiates even though the $\mathrm{Al}$ values are high. Results from these dredged samples also suggest that volcanic activity ceased by the end of the Oligocene.

Table 1. Two analyses of typical basaltic andesites from IGCP Project “Ophiolites," Palau-Kyushu Ridge, 1977.

\begin{tabular}{|c|c|c|c|c|c|c|c|c|c|c|c|}
\hline Samples & $\mathrm{SiO}_{2}$ & $\mathrm{TiO}_{2}$ & & $\mathrm{Al}_{2} \mathrm{O}_{3}$ & $\mathrm{FeO}^{\mathrm{a}}$ & $\begin{array}{l}\text { Mnc } \\
\text { (wt. ? }\end{array}$ & & $\mathrm{MgO}$ & $\mathrm{CaO}$ & $\mathrm{Na}_{2} \mathrm{O}$ & $\mathrm{K}_{2} \mathrm{O}$ \\
\hline \multirow{3}{*}{$\begin{array}{l}\text { Site } 1396 \\
\text { (Rock 1-2) } \\
\text { Site 1397 } \\
\text { (Rock 2-1) }\end{array}$} & 52.3 & 1.10 & & 19.4 & 9.44 & 0.13 & & 2.73 & 8.17 & 3.64 & 1.00 \\
\hline & 54.2 & 1.15 & & 19.0 & 8.99 & 0.22 & & 2.50 & 8.50 & 3.51 & 0.45 \\
\hline & B & $\mathrm{Ba}$ & F & $\mathbf{L i}$ & $\underset{(\mathrm{ppm})}{\mathrm{Sr}}$ & $\mathrm{Zn}$ & $\mathrm{Cu}$ & Co & $\mathrm{Ni}$ & $\mathrm{Cr}$ & \\
\hline $\begin{array}{l}\text { Site } 1396 \\
\text { (Rock 1-2) }\end{array}$ & $<10$ & 70 & 400 & 21.5 & 340 & 173 & 190 & 100 & 45 & 320 & \\
\hline $\begin{array}{l}\text { Site } 1397 \\
\text { (Rock 2-1) }\end{array}$ & $<10$ & 40 & 200 & 4.5 & 270 & 123 & 85 & 100 & 40 & 260 & \\
\hline
\end{tabular}

${ }^{\mathrm{a}} \mathrm{FeO} *$ stands for total $\mathrm{Fe}$ expressed as $\mathrm{FeO}$. 
Studies of volcaniclastic debris drilled at DSDP Hole 296 (Ingle, Karig, et al., 1975) indicate that major volcanism with an accumulation rate of $140 \mathrm{~m} / \mathrm{m}$.y. waned during the late Oligocene on the northern part of the Palau-Kyushu Ridge. Here abundant pyroclastic debris also indicates a shallow-marine or subaerial origin of the volcanic material; the Leg 31 shipboard scientists theorized that the Palau-Kyushu Ridge was an active arc that became inactive and subsequently submerged as the Shikoku and Parece Vela Basins began to form. Magnetic anomaly identification in the Shikoku Basin (Watts and Weissel, 1975; Shih, in press) suggests that $25 \mathrm{~m} . \mathrm{y}$. ago, at the time of cessation of volcanism at Site 296, the Shikoku Basin began to open; this was some 5 m.y. after cessation of volcanism at Site 448 and the initial opening of the Parece Vela Basin.

\section{METHODS}

Analytic methods used in this study include atomic-absorption spectrophotometry (AAS), instrumental neutron-activation analysis (INAA), $x$-ray fluorescence (XRF), electron-microprobe analysis, and a variety of colorimetric techniques. A Perkin-Elmer model 306 at Texas A\&M was used for major-element analyses by AAS with the following precisions in one standard deviation of the oxide: $\mathrm{Al}_{2} \mathrm{O}_{3}=$ $3.5 \%, \mathrm{FeO}^{*}=2.2 \%, \mathrm{MgO}=2.0 \%, \mathrm{CaO}=2.0 \%, \mathrm{Na}_{2} \mathrm{O}=1.2 \%$, $\mathrm{K}_{2} \mathrm{O}=1.4 \%$ and $\mathrm{MnO}=2.0 \%$ (throughout this chapter, $\mathrm{FeO}^{*}$ is total $\mathrm{Fe}$ expressed as $\mathrm{FeO}$ ). The XRF data were collected at the University of Birmingham as part of the data made available for shipboard scientific use. The ARL-EMX electron microprobe at the University of Texas at Austin was used for probe work on glasses and crystalline phases; one-standard-deviation precision show that $\mathrm{SiO}_{2}=$ $0.7 \%, \mathrm{TiO}_{2}=3.8 \%, \mathrm{Al}_{2} \mathrm{O}_{3}=0.5 \%, \mathrm{FeO}^{*}=1.2 \%, \mathrm{MnO}=7.9 \%$, $\mathrm{MgO}=1.6 \%, \mathrm{CaO}=0.8 \%, \mathrm{Na}_{2} \mathrm{O}=1.7 \%$, and $\mathrm{K}_{2} \mathrm{O}=12.5 \%$. Data reduction was done by a modified Bence-Albee program designed for a Hewlett Packard 9825 (Tiezzi and Scott, in press). The TRIGA reactor, a germanium-lithium (GeLi) detector, and a 4096 channel Canberra-Scorpio analyzer system at Texas A\&M University were used for the INAA studies; one-standard-deviation precisions indicate that $\mathrm{Fe}=1.8 \%, \mathrm{Co}=0.9 \%, \mathrm{Cr}=1.3 \%, \mathrm{Hf}=12 \%, \mathrm{Sc}=$ $0.8 \%, \mathrm{La}=1.5 \%, \mathrm{Ce}=10 \%, \mathrm{Sm}=2.1 \%, \mathrm{Eu}=3.0 \%, \mathrm{~Tb}=10 \%$, $\mathrm{Yb}=10 \%$, and $\mathrm{Lu}=15 \%$. Colorimetric methods were used for whole-rock $\mathrm{SiO}_{2}$ and $\mathrm{TiO}_{2}$ analyses (with a standard deviation of $0.8 \%$ for $\mathrm{SiO}_{2}$ and $6.0 \%$ for $\mathrm{TiO}_{2}$ ).

\section{RESULTS}

Table 2 lists in stratigraphic order preliminary majorelement data on whole-rock samples (by AAS and XRF) and on glasses (by microprobe) for rocks cored at Site 448. Analyses of clasts from volcaniclastic sedimentary units inserted between flows are included in the table. Trace-element data by XRF (see Mattey, et al., this volume) will be referred to in the discussion but will not be reported here. Major-element analyses of individual crystalline phases by microprobe are presented in Table 3 , and the INAA trace-element and rare-earth-element (REE) data are compiled in Table 4.

\section{DISCUSSION}

\section{Petrography}

A clear petrographic distinction between the flows and volcaniclastic sedimentary units of the remnant arcs in the Philippine Sea and the flows of the intervening marginal basins is evident, even without chemical analysis. Detailed petrographic descriptions are given in the
Site 448 chapter for the Palau-Kyushu Ridge, in the Site 451 chapter for the West Mariana Ridge, and in Sites 449 and 450 chapters for the Parece Vela Basin. The stratigraphy of flows and volcaniclastic units in Site 448 are also displayed in figures and discussed in detail in the Site $\mathbf{4 4 8}$ chapter. Only a short summary is given here.

The basalts of the West Philippine Basin basement and the Parece Vela Basin basement are commonly plagioclase-olivine-spinel phyric and relatively nonvesicular; in contrast, the basaltic flows and clasts on the two ridges are commonly plagioclase-clinopyroxene-orthopyroxene phyric and have a pronounced vesicularity (up to $30 \%$ ) typical of shallow, more volatile eruptions expected in island arcs. Although fresh olivine is rare in the remnant-arc units (it is found by probe analysis only in Unit 6), there are altered relic phenocrysts that are interpreted as olivine pseudomorphs in Units $8,11,22$, and 28. A coarsely crystalline clast in Unit 10 (tuffs and volcaniclastic breccias) contains hornblende, plagioclase, quartz, magnetite, and ilmenite but no K-feldspar and probably is a hornblende diorite. This clast, however, has a chemistry (discussed later) characteristic of arc tholeiites. The petrographic data from rocks studied at Site 448 therefore support the contention that they have arc-tholeiite affinities.

Another petrographic feature that distinguishes between ridge and basin volcanic rocks is the degree of alteration. Except for the glass margins of pillow lavas, the basalts on the ridges are severely altered and contain abundant zeolites and clay minerals (see Aldrich et al., this volume). A hydrothermal-metamorphic gradient exists in the flows and volcaniclastic breccias that may reflect a geothermal gradient 7 to 9 times the normal gradient; certainly such a thermal regime is in keeping with an island-arc environment. Although the heat flow in the adjacent back-arc basins is slightly high (Watanabe et al., 1977), there is no indication of abnormal hydrothermal alteration effects upon the basin basalts. Probably the severe alteration of ridge basalts is a function of the high geothermal gradient, the high porosity in the flows, and the numerous permeable volcaniclastic units separating the flows.

We made an attempt, based upon petrographic features, to distinguish among flows, sills, and dikes at Site 448 . We tentatively identified 4 sills (Units 33,35 , 45,47 ) and 6 dikes (Units 26, 27, 28, 37, 49 and 51) from the total of 26 recognized igneous units. As expected from physical models of island-arc construction, the intrusive units are toward the base of the pile, have a distinctly lower vesicularity, and average only 6 meters thick-significantly thinner than the 15-meter-thick flows. There are several examples of clear-cut dike relationships where laminated tuffs and massive volcaniclastic breccias are cut by igneous intrusions at attitudes significantly greater than those of bedded units about and below the igneous unit. In some cases it is possible to identify a unit as a dike, even though contacts are absent, when flow structures of vesicles or phenocrysts are not parallel to bedding of adjacent units. This is particularly useful because of incomplete recovery of core. Sills 
Table 2. Major-element whole-rock and glass chemistry of flows, dikes, sills, and clasts in breccias between flows from Site 448.

\begin{tabular}{|c|c|c|c|c|c|c|c|c|c|c|}
\hline $\begin{array}{l}\text { Samplea } \\
\text { (by unit) }\end{array}$ & Type of Analysis ${ }^{b}$ & $\mathrm{SiO}_{2}$ & $\mathrm{TiO}_{2}$ & $\mathrm{Al}_{2} \mathrm{O}_{3}$ & $\mathrm{FeO}^{*}$ & $\mathrm{MnO}$ & $\mathrm{MgO}$ & $\mathrm{CaO}$ & $\mathrm{Na}_{2} \mathrm{O}$ & $\mathrm{K}_{2} \mathrm{O}$ \\
\hline \multicolumn{11}{|l|}{ Unit 6 (flow) } \\
\hline $448-37-1(32)$ & $\mathrm{x}$ & 43.2 & 1.54 & 14.2 & 13.25 & 0.22 & 4.67 & 10.27 & 2.93 & 0.72 \\
\hline $448-37-1(52)$ & a & 50.8 & 1.52 & 13.5 & 14.85 & 0.29 & 4.27 & 9.03 & 2.58 & 1.37 \\
\hline $448-37-1(52)$ & $\mathrm{p}_{1}$ & 51.6 & 1.44 & 13.0 & 15.58 & $-^{\mathrm{c}}$ & 4.04 & 8.60 & 2.49 & 0.38 \\
\hline $448-37-1(52)$ & $\mathrm{p}_{2}$ & 51.8 & 1.50 & 13.0 & 15.62 & - & 4.02 & 8.60 & 2.49 & 0.38 \\
\hline $448-39-1(75)$ & $a^{a}$ & 50.3 & 1.58 & 12.73 & 14.55 & 0.33 & 4.32 & 8.27 & 2.29 & 1.32 \\
\hline $448-39-1(75)$ & $p_{1}$ & 51.0 & 1.51 & 12.94 & 15.02 & - & 4.04 & 8.84 & 2.46 & 0.42 \\
\hline $448-39-1(75)$ & p2 & 51.0 & 1.43 & 12.88 & 15.24 & - & 4.12 & 8.71 & 2.59 & 0.39 \\
\hline $448-39-1(116)$ & $x$ & 49.4 & 1.64 & 13.8 & 13.58 & 0.22 & 4.64 & 9.96 & 2.90 & 0.79 \\
\hline $448-39-2(32)$ & $\mathrm{x}$ & 49.3 & 1.63 & 14.0 & 13.74 & 0.22 & 4.14 & 9.60 & 3.17 & 0.80 \\
\hline $448-40-1$ (68) & a & 50.7 & 1.57 & 12.9 & 13.59 & 0.29 & 4.39 & 10.20 & 2.55 & 0.59 \\
\hline $448-40-1(82)$ & $\mathrm{x}$ & 49.8 & 1.52 & 12.7 & 13.52 & 0.24 & 4.67 & 8.82 & 2.72 & 1.61 \\
\hline \multicolumn{11}{|l|}{ Unit 8 (flow) } \\
\hline $448-43-1$ (119) & $\mathrm{x}$ & 48.5 & 1.60 & 13.4 & 14.53 & 0.28 & 3.59 & 8.63 & 2.81 & 1.31 \\
\hline $448-43-2(21)$ & a & 51.2 & 1.57 & 13.6 & 14.37 & 0.17 & 4.05 & 8.34 & 2.55 & 0.63 \\
\hline $448-44-1(103)$ & $\mathrm{x}$ & 49.6 & 1.54 & 14.0 & 14.00 & 0.23 & 4.63 & 9.54 & 2.95 & 0.57 \\
\hline \multicolumn{11}{|l|}{ Unit 9 (flow) } \\
\hline $448-47-2(22)$ & a & 50.9 & 1.48 & 14.1 & 13.73 & 0.31 & 3.77 & 8.52 & 2.26 & 1.40 \\
\hline $448-47-2(22)$ & $p_{1}$ & 52.8 & 1.23 & 13.4 & 11.88 & - & 3.14 & 8.05 & 2.38 & 0.38 \\
\hline $448-47-2(22)$ & $\mathrm{p}_{2}$ & 53.0 & 1.32 & 12.7 & 13.99 & - & 3.20 & 7.98 & 2.07 & 0.41 \\
\hline $448-48-1(77)$ & $x$ & 47.9 & 1.60 & 14.6 & 14.61 & 0.23 & 3.24 & 9.45 & 3.11 & 0.86 \\
\hline $448-48-1$ (119) & a & 50.7 & 1.53 & 13.9 & 15.15 & 0.24 & 3.28 & 8.19 & 2.77 & 0.51 \\
\hline $448-48-2(18)$ & a & 50.4 & 1.57 & 13.5 & 14.16 & 0.26 & 3.81 & 8.27 & 2.61 & 0.59 \\
\hline $448-48-2(93)$ & $\mathrm{x}$ & 47.7 & 1.55 & 14.1 & 14.26 & 0.25 & 3.37 & 10.29 & 3.00 & 0.61 \\
\hline $448-48-3(22)$ & $\mathrm{x}$ & 49.8 & 1.52 & 14.3 & 13.94 & 0.23 & 4.48 & 9.53 & 2.85 & 0.68 \\
\hline \multicolumn{11}{|l|}{ Unit 10 (clast) } \\
\hline $448-50-3(80)$ & a & 60.0 & 1.30 & 12.8 & 11.11 & 0.24 & 2.37 & 5.72 & 2.77 & 0.46 \\
\hline $448-50-4(50)$ & a & 47.2 & 1.45 & 16.6 & 11.30 & 0.16 & 4.48 & 7.93 & 3.93 & 1.17 \\
\hline \multicolumn{11}{|l|}{ Unit 11 (flow) } \\
\hline $448-51-4(38)$ & $\mathrm{x}$ & 49.4 & 1.28 & 13.4 & 14.39 & 0.22 & 5.11 & 9.29 & 2.40 & 1.10 \\
\hline $448-52-1(80)$ & $\mathrm{x}$ & 49.2 & 1.21 & 13.9 & 12.66 & 0.24 & 4.56 & 9.69 & 2.80 & 1.14 \\
\hline 448-53-1 (2) & a & 50.6 & 1.27 & 13.6 & 12.43 & 0.26 & 4.33 & 5.29 & 2.41 & 1.23 \\
\hline $448-53-1(2)$ & $p_{1}$ & 51.9 & 1.11 & 13.3 & 13.45 & - & 4.62 & 8.78 & 2.46 & 0.37 \\
\hline $448-53-1(2)$ & $\mathrm{P}_{2}$ & 52.4 & 1.01 & 14.3 & 13.20 & - & 4.48 & 8.91 & 2.62 & 0.33 \\
\hline $448-53-2(58)$ & a & 49.8 & 1.19 & 14.5 & 13.56 & 0.22 & 4.87 & 9.03 & 2.29 & 0.51 \\
\hline $448-53-2(59)$ & $\mathrm{x}$ & 49.2 & 1.15 & 14.7 & 12.12 & 0.20 & 5.19 & 10.23 & 3.33 & 0.47 \\
\hline \multicolumn{11}{|l|}{ Unit 12 (clast) } \\
\hline $448-55-1(110)$ & a & 50.6 & 1.05 & 14.5 & 6.39 & 0.11 & 2.29 & 4.32 & 4.53 & 4.25 \\
\hline $448-57-1(33)$ & a & 48.7 & 0.93 & 16.1 & 10.81 & 0.07 & 6.56 & 9.56 & 2.57 & 1.09 \\
\hline $448-57-2(16)$ & a & 49.2 & 1.21 & 16.7 & 12.28 & 0.15 & 4.89 & 7.83 & 2.95 & 1.36 \\
\hline $448-57-2(102)$ & $\mathrm{x}$ & 48.9 & 0.81 & 17.1 & 10.47 & 0.22 & 5.37 & 10.56 & 2.45 & 0.56 \\
\hline $448-58-2(58)$ & a & 50.3 & 0.97 & 14.3 & 12.85 & 0.28 & 5.29 & 9.27 & 2.34 & 0.50 \\
\hline $448-58-2(49)$ & a & 51.0 & 1.30 & 14.2 & 11.36 & 0.27 & 3.92 & 8.38 & 2.64 & 1.79 \\
\hline \multicolumn{11}{|l|}{ Unit 13 (flow) } \\
\hline $448-58-3(77)$ & $\mathrm{x}$ & 49.9 & 1.12 & 14.5 & 11.29 & 0.19 & 4.52 & 9.24 & 3.39 & 1.65 \\
\hline $448-58-3(113)$ & a & 53.7 & 1.04 & 13.2 & 12.16 & 0.17 & 4.32 & 7.72 & 2.42 & 1.57 \\
\hline $448-58-3(113)$ & $\mathrm{p}_{1}$ & 55.4 & 1.14 & 13.6 & 12.37 & - & 3.65 & 8.35 & 2.43 & 0.44 \\
\hline 448-58-3 (113) & $\mathrm{p}_{2}$ & 54.1 & 1.09 & 13.3 & 12.60 & - & 3.64 & 8.11 & 2.80 & 0.52 \\
\hline $448-58-4(138)$ & $\mathrm{x}$ & 48.0 & 1.02 & 14.2 & 10.24 & 0.17 & 3.88 & 12.74 & 4.56 & 1.20 \\
\hline $448-59-1(62)$ & a & 52.9 & 1.16 & 13.5 & 11.70 & 0.21 & 5.25 & 8.19 & 2.79 & 1.38 \\
\hline $448-59-1(62)$ & $p_{1}$ & 55.4 & 1.12 & 13.3 & 12.43 & - & 3.67 & 8.11 & 2.53 & 0.49 \\
\hline 448-59-1 (62) & $\mathrm{p}_{2}$ & 55.6 & 1.17 & 13.5 & 12.53 & - & 3.69 & 8.45 & 2.74 & 0.45 \\
\hline $448-59-2(61)$ & $x$ & 50.7 & 1.09 & 16.8 & 10.81 & 0.19 & 4.47 & 9.80 & 2.98 & 0.83 \\
\hline 448-59-3 (51) & $\mathrm{p}_{1}$ & 54.5 & 1.09 & 14.2 & 12.66 & - & 3.66 & 7.83 & 2.40 & 0.45 \\
\hline $448-59-3(51)$ & $\mathrm{p}_{2}$ & 54.8 & 1.16 & 13.2 & 12.69 & - & 3.70 & 8.07 & 2.23 & 0.44 \\
\hline $448-59-3(74)$ & $\mathrm{x}$ & 50.0 & 1.10 & 16.4 & 10.48 & 0.17 & 4.28 & 10.27 & 3.58 & 1.19 \\
\hline $448-61-3(55)$ & $\mathrm{x}$ & 50.2 & 1.12 & 15.0 & 11.01 & 0.15 & 6.30 & 8.84 & 3.44 & 1.41 \\
\hline \multicolumn{11}{|l|}{ Unit 13 (flow) } \\
\hline $448 \mathrm{~A}-15-1(30)$ & a & 52.6 & 1.03 & 15.15 & 10.96 & 0.16 & 4.05 & 8.41 & 2.73 & 1.49 \\
\hline $448 \mathrm{~A}-15-1(30)$ & $\mathrm{p}_{1}$ & 54.9 & 1.11 & 13.5 & 12.08 & - & 3.56 & 8.26 & 2.23 & 0.41 \\
\hline $448 \mathrm{~A}-15-1(30)$ & p2 & 54.7 & 1.09 & 13.8 & 12.22 & - & 3.55 & 8.03 & 1.94 & 0.44 \\
\hline $448 \mathrm{~A}-15-1$ (123) & $\mathrm{x}$ & 49.4 & 1.11 & 15.9 & 11.02 & 0.20 & 4.68 & 10.12 & 3.47 & 0.94 \\
\hline $448 \mathrm{~A}-15-3$ (143) & $\mathrm{x}$ & 50.2 & 1.08 & 16.4 & 10.70 & 0.18 & 4.02 & 10.16 & 3.16 & 1.39 \\
\hline $448 \mathrm{~A}-16-2(71)$ & a & 52.2 & 1.06 & 15.7 & 11.36 & 0.17 & 4.07 & 8.16 & 2.38 & 1.26 \\
\hline $448 \mathrm{~A}-16-2(71)$ & $\mathrm{p}_{1}$ & 55.2 & 1.12 & 13.5 & 12.30 & - & 3.69 & 8.18 & 2.70 & 0.49 \\
\hline $448 \mathrm{~A}-16-2(71)$ & $\mathrm{p}_{2}$ & 55.2 & 1.02 & 13.4 & 12.40 & - & 3.71 & 8.20 & 2.79 & 0.44 \\
\hline
\end{tabular}


Table 2. (Continued),

\begin{tabular}{|c|c|c|c|c|c|c|c|c|c|c|}
\hline $\begin{array}{l}\text { Samplea } \\
\text { (by unit) }\end{array}$ & Type of Analysis ${ }^{b}$ & $\mathrm{SiO}_{2}$ & $\mathrm{TiO}_{2}$ & $\mathrm{Al}_{2} \mathrm{O}_{3}$ & $\mathrm{FeO}^{*}$ & $\mathrm{MnO}$ & $\mathrm{MgO}$ & $\mathrm{CaO}$ & $\mathrm{Na}_{2} \mathrm{O}$ & $\mathrm{K}_{2} \mathrm{O}$ \\
\hline \multicolumn{11}{|l|}{ Unit 14 (flow) } \\
\hline $448 \mathrm{~A}-16-3(10)$ & $x$ & 50.0 & 1.09 & 15.9 & 11.00 & 0.19 & 4.96 & 9.79 & 3.17 & 1.05 \\
\hline $448 \mathrm{~A}-17-1(87)$ & $\mathrm{x}$ & 49.7 & 1.15 & 15.5 & 11.49 & 0.20 & 4.11 & 9.72 & 3.00 & 1.33 \\
\hline $448 \mathrm{~A}-18-1$ (77) & $x$ & 50.8 & 1.12 & 14.5 & 11.45 & 0.21 & 4.14 & 8.93 & 3.14 & 1.82 \\
\hline $448 \mathrm{~A}-18-1(112)$ & $p_{1}$ & 55.4 & 1.11 & 13.4 & 12.63 & - & 3.66 & 8.33 & 2.64 & 0.45 \\
\hline $448 \mathrm{~A}-18-1(112)$ & $\mathrm{p}_{2}$ & 55.4 & 1.14 & 13.5 & 12.63 & - & 3.62 & 8.25 & 2.42 & 0.47 \\
\hline $448 \mathrm{~A}-18-1$ (112) & $\mathrm{p}_{3}$ & 55.2 & 1.13 & 13.4 & 12.50 & - & 3.65 & 8.30 & 2.72 & 0.47 \\
\hline $448 \mathrm{~A}-18-1(112)$ & $\mathrm{p}_{4}$ & 55.9 & 1.20 & 13.7 & 12.66 & - & 3.65 & 8.29 & 2.67 & 0.50 \\
\hline $448 \mathrm{~A}-20-1(118)$ & $x$ & 51.2 & 1.08 & 14.2 & 12.29 & 0.21 & 4.66 & 8.32 & 2.99 & 2.14 \\
\hline $448 \mathrm{~A}-20-4(4)$ & $x$ & 49.8 & 1.29 & 14.0 & 11.35 & 0.21 & 4.41 & 8.88 & 3.04 & 1.66 \\
\hline \multicolumn{11}{|l|}{ Unit 15 (clast) } \\
\hline $448 \mathrm{~A}-21-1(56)$ & a & 51.8 & 1.03 & 14.5 & 10.12 & 0.13 & 2.97 & 7.93 & 3.34 & 0.88 \\
\hline $448 \mathrm{~A}-21-1(77)$ & a & 50.4 & 1.17 & 14.6 & 11.51 & 0.59 & 4.09 & 7.98 & 3.05 & 1.19 \\
\hline $448 \mathrm{~A}-22-1(7)$ & a & 49.9 & 1.21 & 14.6 & 11.13 & 0.07 & 3.66 & 7.76 & 3.47 & 1.25 \\
\hline $448 \mathrm{~A}-25-1(23)$ & $x$ & 49.8 & 1.28 & 14.1 & 11.35 & 0.21 & 6.21 & 9.32 & 3.52 & 0.63 \\
\hline \multicolumn{11}{|l|}{ Unit 16 (flow) } \\
\hline $448 \mathrm{~A}-26-2(32)$ & a & 47.5 & 1.31 & 15.8 & 11.73 & 0.21 & 6.04 & 9.07 & 3.56 & 1.10 \\
\hline $448 A-26-2(60)$ & $x$ & 47.7 & 1.22 & 13.2 & 11.72 & 0.37 & 6.47 & 9.93 & 3.48 & 1.63 \\
\hline \multicolumn{11}{|l|}{ Unit 18 (flow) } \\
\hline $448 \mathrm{~A}-28-1(52)$ & $\mathrm{x}$ & 49.0 & 1.13 & 15.4 & 12.6 & 0.20 & 7.02 & 6.14 & 2.44 & 1.18 \\
\hline $448 \mathrm{~A}-28-1(96)$ & a & 48.2 & 1.01 & 12.9 & 11.23 & 0.26 & 9.22 & 6.38 & 2.58 & 2.03 \\
\hline \multicolumn{11}{|l|}{ Unit 20 (flow) } \\
\hline $448 \mathrm{~A}-32-1(47)$ & $\mathrm{x}$ & 51.2 & 1.41 & 13.4 & 12.66 & 0.18 & 6.19 & 8.29 & 3.10 & 1.47 \\
\hline $448 \mathrm{~A}-33-1$ (134) & a & 49.6 & 1.36 & 14.6 & 14.17 & 0.26 & 5.87 & 8.80 & 2.72 & 0.67 \\
\hline $448 \mathrm{~A}-33-2(6)$ & a & 49.7 & 1.33 & 15.2 & 12.52 & 0.28 & 5.47 & 8.75 & 2.74 & 0.65 \\
\hline \multicolumn{11}{|l|}{ Unit 22 (flow) } \\
\hline $448 \mathrm{~A}-36-5(53)$ & a & 49.9 & 1.01 & 14.0 & 10.79 & 0.04 & 6.67 & 10.07 & 2.06 & 0.16 \\
\hline $448 \mathrm{~A}-36-7(130)$ & & 50.6 & 0.95 & 13.5 & 11.56 & 0.26 & 8.18 & 10.33 & 2.62 & 0.16 \\
\hline \multicolumn{11}{|l|}{ Unit 23 (flow) } \\
\hline $448 \mathrm{~A}-37-2(141)$ & a & 50.9 & 1.48 & 12.8 & 12.29 & 0.24 & 5.80 & 7.29 & 2.32 & 1.03 \\
\hline $448 \mathrm{~A}-37-2(145)$ & $x$ & 50.0 & 1.63 & 12.6 & 13.07 & 0.22 & 6.43 & 9.01 & 2.76 & 1.16 \\
\hline $448 \mathrm{~A}-38-1$ (99) & a & 49.6 & 1.51 & 12.0 & 12.06 & 0.27 & 6.35 & 6.40 & 1.93 & 3.73 \\
\hline \multicolumn{11}{|l|}{ Unit 24 (flow) } \\
\hline $448 \mathrm{~A}-39-2(140)$ & $\begin{array}{l}x \\
\mathrm{a}\end{array}$ & 49.8 & 1.36 & 13.2 & 12.83 & 0.22 & 5.61 & $\begin{array}{l}9.21 \\
8,2.3\end{array}$ & $\begin{array}{l}4.55 \\
2.49\end{array}$ & 1.11 \\
\hline 448A-40-1 (33) & & 50.2 & 1.31 & 14.9 & 13.14 & 0.48 & 4.71 & & & 0.57 \\
\hline $448 \mathrm{~A}-41-1$ (17) & $\begin{array}{l}\mathrm{x} \\
\mathrm{a}\end{array}$ & 49.7 & 1.25 & 13.6 & 12.10 & 0.21 & 6.70 & 9.15 & 2.64 & 1.28 \\
\hline $448 \mathrm{~A}-41-1(90)$ & & 50.3 & 1.30 & 14.2 & 11.22 & 0.27 & 4.31 & 8.44 & 2.53 & 1.75 \\
\hline \multicolumn{11}{|l|}{ Unit 27 (dike) } \\
\hline $448 \mathrm{~A}-41-2(12)$ & $a_{1}$ & 49.5 & 1.28 & 13.4 & 11.56 & 0.28 & 6.44 & 9.08 & 2.28 & 0.45 \\
\hline $448 \mathrm{~A}-41-2(12)$ & $a_{2}$ & 50.0 & 1.27 & 13.2 & 11.72 & 0.27 & 6.31 & 8.44 & 2.53 & 1.75 \\
\hline $448 \mathrm{~A}-41-2(58)$ & $x$ & 49.9 & 1.14 & 12.6 & 12.92 & 0.20 & 7.46 & 9.02 & 2.79 & 0.81 \\
\hline $448 \mathrm{~A}-41-4(4)$ & $\mathrm{x}$ & 50.5 & 1.43 & 13.1 & 12.74 & 0.22 & 7.26 & 8.94 & 3.02 & 0.50 \\
\hline $448 \mathrm{~A}-41-4(16)$ & a & 50.4 & 1.30 & 13.4 & 12.56 & 0.28 & 7.44 & 8.08 & 2.82 & 0.45 \\
\hline \multicolumn{11}{|l|}{ Unit 31 (flow) } \\
\hline $\begin{array}{l}448 \mathrm{~A}-44-1(34) \\
448 \mathrm{~A}-44-2(55)\end{array}$ & $\begin{array}{l}a \\
x\end{array}$ & $\begin{array}{l}54.1 \\
54.5\end{array}$ & $\begin{array}{l}1.32 \\
1.49\end{array}$ & $\begin{array}{l}13.9 \\
13.0\end{array}$ & 11.77 & $\begin{array}{l}0.34 \\
0.22\end{array}$ & $\begin{array}{l}5.86 \\
5.85\end{array}$ & $\begin{array}{l}6.64 \\
7.55\end{array}$ & $\begin{array}{l}2.36 \\
2.94\end{array}$ & $\begin{array}{l}0.12 \\
0.36\end{array}$ \\
\hline $\begin{array}{l}448 \mathrm{~A}-44-2(55) \\
448 \mathrm{~A}-45-1(43)\end{array}$ & $\hat{a}$ & $\begin{array}{l}54.5 \\
53.6\end{array}$ & 1.41 & $\begin{array}{l}13.0 \\
13.4\end{array}$ & $\begin{array}{l}11.63 \\
11.77\end{array}$ & 0.16 & 5.26 & 7.50 & 2.40 & 0.17 \\
\hline $448 \mathrm{~A}-45-2$ (13) & a & 54.4 & 1.33 & 13.8 & 11.58 & 0.10 & 4.50 & 8.02 & 2.52 & 0.18 \\
\hline $448 \mathrm{~A}-45-2(22)$ & $\mathrm{x}$ & 52.1 & 1.36 & 13.9 & 11.26 & 0.19 & 6.16 & 9.45 & 2.46 & 0.20 \\
\hline $448 \mathrm{~A}-46-2(82)$ & $\mathrm{x}$ & 54.7 & 1.21 & 13.5 & 11.87 & 0.18 & 5.71 & 7.75 & 2.81 & 0.34 \\
\hline \multicolumn{11}{|l|}{ Unit 33 (sill) } \\
\hline $\begin{array}{l}448 A-47-1(72) \\
448 A-47-1(72)\end{array}$ & $\begin{array}{l}\mathrm{a} \\
\mathrm{a}\end{array}$ & 53.5 & 1.38 & 13.8 & 12.97 & 0.17 & 4.32 & 7.08 & 2.70 & 0.30 \\
\hline $\begin{array}{l}448 A-47-1(72) \\
448 A-47-2(108)\end{array}$ & $\begin{array}{l}\mathrm{a} \\
\mathrm{x}\end{array}$ & $\begin{array}{l}53.9 \\
48.3\end{array}$ & 1.42 & 13.4 & 12.99 & 0.12 & 4.07 & 6.18 & $\begin{array}{l}3.61 \\
2.92\end{array}$ & 0.56 \\
\hline $448 \mathrm{~A}-47-2(108)$ & $\mathrm{x}$ & 48.3 & 1.55 & 13.9 & 14.32 & 0.25 & 3.84 & 9.52 & 2.92 & 0.79 \\
\hline $448 \mathrm{~A}-47-2(106)$ & $\mathrm{x}$ & 55.0 & 1.23 & 13.5 & 12.40 & 0.18 & 5.59 & 7.74 & 2.84 & 0.32 \\
\hline $448 \mathrm{~A}-47-3(138)$ & $a_{1}$ & 54.7 & 1.36 & 13.3 & 12.50 & 0.10 & 4.37 & 7.06 & 2.97 & 0.28 \\
\hline $448 \mathrm{~A}-47-3(138)$ & $a_{2}$ & 53.2 & 1.58 & 13.5 & 12.97 & 0.08 & 3.42 & 6.75 & 2.78 & 0.36 \\
\hline $448 \mathrm{~A}-47-3(138)$ & $a_{3}$ & 54.1 & 1.27 & 15.2 & 12.01 & 0.14 & 4.36 & 6.92 & 2.76 & 0.30 \\
\hline Unit 35 (sill) & & & & & & & & & & \\
\hline $448 \mathrm{~A}-50-1$ (47) & a & 53.0 & 1.37 & 13.6 & 12.98 & 0.32 & 6.02 & 7.58 & 2.75 & 0.32 \\
\hline $448 \mathrm{~A}-50-1$ (95) & a & 53.7 & 1.42 & 12.4 & 13.24 & 0.36 & 5.04 & 8.22 & 2.26 & 0.28 \\
\hline $448 \mathrm{~A}-50-1$ (99) & a & 53.4 & 1.39 & 12.9 & 12.24 & 0.34 & 5.33 & 7.71 & 2.47 & 0.33 \\
\hline $448 \mathrm{~A}-50-2(35)$ & $\mathrm{x}$ & 53.3 & 1.45 & 12.8 & 12.49 & 0.24 & 5.44 & 8.33 & 2.55 & 0.30 \\
\hline
\end{tabular}


Table 2. (Continued).

\begin{tabular}{|c|c|c|c|c|c|c|c|c|c|c|}
\hline $\begin{array}{l}\text { Sample }{ }^{\mathrm{a}} \\
\text { (by unit) }\end{array}$ & Type of Analysis ${ }^{b}$ & $\mathrm{SiO}_{2}$ & $\mathrm{TiO}_{2}$ & $\mathrm{Al}_{2} \mathrm{O}_{3}$ & $\mathrm{FeO}^{*}$ & $\mathrm{MnO}$ & $\mathrm{MgO}$ & $\mathrm{CaO}$ & $\mathrm{Na}_{2} \mathrm{O}$ & $\mathrm{K}_{2} \mathrm{O}$ \\
\hline \multicolumn{11}{|l|}{ Unit 37 (dike) } \\
\hline 448A-51-1 (85) & a & 54.3 & 1.33 & 12.3 & 12.87 & 0.29 & 4.86 & 7.30 & 2.21 & 0.29 \\
\hline $448 \mathrm{~A}-51-1(85)$ & $\mathrm{p}_{1}$ & 42.4 & 1.79 & 12.0 & 19.37 & - & 5.22 & 8.20 & 1.43 & 0.38 \\
\hline $448 A-51-1(85)$ & $\mathrm{p}_{2}$ & 42.9 & 1.86 & 11.7 & 20.73 & - & 6.09 & 8.20 & 1.37 & 0.42 \\
\hline $448 \mathrm{~A}-51-1(85)$ & $\mathrm{p}_{3}$ & 54.2 & 1.31 & 12.7 & 14.42 & - & 3.23 & 7.67 & 2.63 & 0.42 \\
\hline $448 \mathrm{~A}-51-1(85)$ & $\mathrm{p}_{4}$ & 54.4 & 1.31 & 13.0 & 14.11 & - & 3.33 & 8.12 & 2.89 & 0.34 \\
\hline $448 \mathrm{~A}-51-2(134)$ & $\mathrm{x}$ & 54.8 & 1.27 & 12.8 & 12.27 & 0.20 & 5.97 & 7.69 & 2.58 & 0.25 \\
\hline $448 \mathrm{~A}-51-3(135)$ & a & 54.2 & 1.34 & 12.1 & 11.38 & 0.17 & 5.41 & 7.36 & 2.53 & 0.34 \\
\hline \multicolumn{11}{|l|}{ Unit 39 (flow) } \\
\hline 448A-52-1 (77) & $\mathrm{x}$ & 51.1 & 1.48 & 12.9 & 12.92 & 0.22 & 6.48 & 8.76 & 2.48 & 0.10 \\
\hline $448 \mathrm{~A}-52-2(128)$ & a & 51.6 & 1.51 & 12.6 & 12.91 & 0.09 & 6.36 & 7.82 & 2.70 & 0.41 \\
\hline \multicolumn{11}{|l|}{ Unit 41 (flow) } \\
\hline $448 \mathrm{~A}-54-3(81)$ & a & 53.8 & 1.32 & 13.5 & 11.22 & 0.18 & 4.29 & 8.83 & 2.53 & 0.27 \\
\hline $448 \mathrm{~A}-54-3(115)$ & $x$ & 54.1 & 1.25 & 13.6 & 11.71 & 0.21 & 5.78 & 8.58 & 2.61 & 0.31 \\
\hline $448 \mathrm{~A}-56-1(31)$ & a & 54.3 & 1.28 & 13.5 & 11.46 & 0.10 & 4.70 & 8.62 & 2.56 & 0.33 \\
\hline \multicolumn{11}{|l|}{ Unit 43 (flow) } \\
\hline $448 \mathrm{~A}-57-1(96)$ & $\mathrm{x}$ & 55.2 & 1.28 & 12.9 & 12.12 & 0.18 & 6.01 & 7.76 & 2.63 & 0.37 \\
\hline \multicolumn{11}{|l|}{ Unit 47 (sill) } \\
\hline $448 \mathrm{~A}-59-2(85)$ & $\mathrm{x}$ & 51.5 & 1.68 & 13.5 & 11.95 & 0.21 & 5.80 & 9.54 & 2.92 & 0.38 \\
\hline $448 \mathrm{~A}-59-2(126)$ & a & 53.2 & 1.52 & 13.3 & 11.32 & 0.18 & 5.33 & 7.36 & 2.50 & 0.19 \\
\hline \multicolumn{11}{|l|}{ Unit 49 (dike) } \\
\hline $448 \mathrm{~A}-62-1(60)$ & $a_{1}$ & 52.7 & 1.57 & 12.1 & 12.48 & 0.12 & 5.09 & 9.51 & 2.46 & 0.25 \\
\hline $448 \mathrm{~A}-62-1(60)$ & $a_{2}$ & 53.0 & 1.63 & 12.8 & 12.94 & 0.18 & 5.01 & 7.90 & 2.66 & 0.42 \\
\hline $448 \mathrm{~A}-62-2(7)$ & a & 53.6 & 1.49 & 12.4 & 9.80 & 0.10 & 5.58 & 9.55 & 2.59 & 0.14 \\
\hline \multicolumn{11}{|l|}{ Unit 51 (dike) } \\
\hline 448A-65-1 (81) & $\mathrm{x}$ & 52.2 & 1.17 & 13.3 & 12.07 & 0.22 & 5.91 & 9.04 & 2.63 & 0.19 \\
\hline $448 \mathrm{~A}-65-2(130)$ & a & 52.6 & 1.22 & 13.0 & 12.52 & 0.20 & 5.53 & 8.23 & 2.50 & 0.20 \\
\hline $448 \mathrm{~A}-66-2(125)$ & $a_{1}$ & 51.9 & 1.26 & 13.6 & 11.45 & 0.05 & 5.41 & 8.61 & 2.37 & 0.23 \\
\hline $448 \mathrm{~A}-66-2(125)$ & $a_{2}$ & 52.7 & 1.31 & 13.2 & 11.99 & 0.08 & 5.43 & 8.25 & 3.24 & 0.38 \\
\hline
\end{tabular}

a Sample designations give the hole number-core number-section, and center of the sample interval (in parentheses)

in stratigraphic order. Unit numbers are the same as those in the Site 448 chapter.

$\mathrm{b} x \doteq \mathrm{XRF}$ data collected at the University of Birmingham by John Tarney, David Mattey, and Nicholas Marsh for use by shipboard scientists on Leg 59; $\mathrm{a}=$ atomic absorption and colorimetric methods; $\mathrm{p}=$ glass microprobe data; subscripts 1, 2, 3, and 4 refer to different areas analyzed by probe or by atomic absorption and colorimetric methods at the same interval.

c Dash (-) indicates that no analysis was made.

are more difficult to recognize. Where upper and lower contacts show abnormally thin quenched margins and usually an absence of glass, sills may be expected. Some intrusive units, however, can apparently form glassy margins against cool country rock, as shown by the thin glassy margin of the basaltic dike of Unit 37 . In one case, the chilled margins of a sill have been symmetrically deformed to produce drag features on the top and bottom, presumably created by repeated injections of magma. In another case, a symmetrical differentiation of plagioclase laths at the top and bottom of a sill suggests that symmetrical boundary-flow conditions existed. In either case it is difficult to envision mechanisms that might create such symmetry in an extrusive flow.

\section{Chemistry}

Whole-rock chemistry (Table 2) of nonglass flow interiors by AAS and XRF shows a high degree of scatter; attempts to characterize the units using Kuno's (1965) $\mathrm{SiO}_{2}$-alkali $\left(\mathrm{Na}_{2} \mathrm{O}+\mathrm{K}_{2} \mathrm{O}\right)$ plot suggested that alkaliolivine, high-alumina, and tholeiitic basaltic magmas all coexisted in one 600-meter sequence of lavas. This unlikely possibility was supported by Miyashiro's (1974) $\mathrm{SiO}_{2}-\mathrm{FeO} * / \mathrm{MgO}$ plots, in which trends of analyses fell into both the calc-alkalic and the tholeiitic fields. The AFM plot of these data is somewhat more discriminatory (Fig. 1) but still shows considerable scatter between the Skaergaard tholeiitic trend and those of Asama and Amagi calc-alkalic trends. If only probe analyses of fresh glasses are plotted, however, a tight group near the Skaergaard trend line is produced (Fig. 2) with an elongation toward the alkali apex. An enlargement of that group (Fig. 3) from $5 \%$ to $25 \%$ alkalis, $60 \%$ to $80 \% \mathrm{FeO}^{*}$, and $15 \%$ to $35 \% \mathrm{MgO}$ shows that each volcanic unit has very distinct alkali-enrichment trends. Such trends might be created by two scales of crystal fractionation: (1) crystal fractionation on a unit-wide scale or (2) local crystal fractionation within the quenched pillow margins. The tholeiitic character of these rocks is further confirmed by the Kuno plot (Fig. 4) and the Miyashiro plot (Fig. 5) of glass compositions. Obviously the quenched glassy margins of the pillows represent a reasonable approximation of a closed system even in otherwise extensively altered rocks (Scott and Hajash, 1976).

Comparisons among the tholeiitic mid-ocean-ridge basalts, arc tholeiites, calc-alkalic basalts, and the drilled Palau-Kyushu Ridge rocks using both major- 
Table 3. Major-element chemistry of phases within flows, sills, and dikes from Site 448.

\begin{tabular}{|c|c|c|c|c|c|c|c|c|c|c|c|}
\hline Sample ${ }^{a}$ & Unit & $\mathrm{SiO}_{2}$ & $\mathrm{TiO}_{2}$ & $\mathrm{Al}_{2} \mathrm{O}_{3}$ & $\mathrm{FeO}^{*}$ & $\mathrm{MnO}$ & $\mathrm{MgO}$ & $\mathrm{CaO}$ & $\mathrm{Na}_{2} \mathrm{O}$ & $\mathrm{K}_{2} \mathrm{O}$ & $\mathrm{En} / \mathrm{Fs} / \mathrm{Wo}^{\mathrm{b}}$ \\
\hline \multicolumn{12}{|c|}{ Clinopyroxenes } \\
\hline $448-37-1(52)_{1}$ & 6 & 50.9 & 0.58 & 3.23 & 15.13 & $-e^{e}$ & 18.89 & 15.45 & 0.20 & 0.07 & $49 / 22 / 29$ \\
\hline $448-37-1(52)_{2}$ & 6 & - & - & - & 13.75 & - & 17.98 & 17.60 & 0.10 & 0.05 & $47 / 20 / 33$ \\
\hline $448-39-1(75)_{1}$ & 6 & 50.5 & 0.58 & 2.96 & 13.93 & - & 16.91 & 16.61 & 0.14 & 0.02 & $46 / 21 / 33$ \\
\hline $448-39-1(75)_{2}$ & 6 & 51.1 & 0.56 & 3.12 & 17.08 & - & 18.01 & 13.39 & 0.09 & 0.06 & $48 / 26 / 26$ \\
\hline $448-39-1(75)_{3}$ & 6 & 50.6 & 0.56 & 3.07 & 14.57 & - & 14.51 & 15.28 & 0.19 & 0.11 & $43 / 24 / 33$ \\
\hline $448-39-1(75)_{4}$ & 6 & 52.3 & 1.43 & 3.30 & 14.96 & - & 15.88 & 14.41 & 0.16 & 0.03 & $46 / 24 / 30$ \\
\hline $448-53-1(2)$ & 10 & 50.6 & 0.46 & 3.55 & 12.93 & - & 16.76 & 15.49 & 0.11 & 0.02 & $48 / 21 / 32$ \\
\hline $448-58-3(113)_{1}$ & 12 & 52.2 & 0.36 & 4.48 & 7.05 & - & 16.91 & 20.56 & 0.09 & 0.02 & $47 / 11 / 42$ \\
\hline $448-58-3(113) 2$ & 12 & 51.8 & 0.34 & 2.24 & 11.98 & - & 16.11 & 17.95 & 0.15 & 0.04 & $45 / 19 / 36$ \\
\hline $448-59-1(62)$ & 12 & 52.4 & 0.31 & 2.20 & 10.02 & - & 16.88 & 18.15 & 0.30 & 0.05 & $47 / 16 / 37$ \\
\hline $448 \mathrm{~A}-18-1(112)_{1}$ & 14 & 51.1 & 0.32 & 1.98 & 11.51 & - & 16.04 & 18.27 & 0.15 & 0.01 & $45 / 18 / 37$ \\
\hline $448 \mathrm{~A}-18-1(112)_{2}$ & 14 & 53.1 & 0.34 & 2.05 & 11.29 & - & 16.07 & 18.69 & 0.16 & 0.03 & $45 / 18 / 37$ \\
\hline $448 \mathrm{~A}-4 \mathrm{I}-4(16)_{1}$ & 27 & 51.1 & 0.36 & 2.09 & 11.37 & 0.03 & 17.13 & 18.22 & 0.24 & 0.00 & $46 / 18 / 36$ \\
\hline $448 \mathrm{~A}-41-4(16)_{2}$ & 27 & 50.7 & 0.31 & 2.03 & 9.12 & 0.28 & 17.60 & 19.74 & 0.21 & 0.00 & $48 / 14 / 38$ \\
\hline $448 \mathrm{~A}-50-1(99)_{1}$ & 35 & 53.0 & 0.42 & 2.37 & 10.75 & 0.33 & 16.84 & 19.17 & 0.23 & 0.01 & $46 / 16 / 38$ \\
\hline 448 A-50-1 $(99)_{2}$ & 35 & 53.3 & 0.30 & 2.32 & 10.41 & 0.30 & 16.86 & 19.19 & 0.20 & 0.00 & $46 / 16 / 38$ \\
\hline $448 A-51-1(85)^{2}$ & 37 & 49.4 & 0.79 & 3.89 & 16.83 & 0.54 & 16.57 & 14.55 & 0.18 & 0.05 & $45 / 26 / 29$ \\
\hline $448 \mathrm{~A}-56-1(31)_{1}$ & 41 & 51.4 & 0.35 & 2.49 & 9.43 & 0.26 & 16.43 & 19.76 & 0.19 & 0.01 & $46 / 15 / 39$ \\
\hline $448 \mathrm{~A}-56-1(31)_{2}$ & 41 & 51.8 & 0.34 & 2.31 & 10.00 & 0.29 & 16.67 & 20.22 & 0.19 & 0.03 & $45 / 15 / 40$ \\
\hline 448 A-59-2 (126) & 47 & 51.5 & 0.35 & 2.10 & 10.82 & 0.29 & 16.90 & 18.95 & 0.22 & 0.00 & $46 / 17 / 37$ \\
\hline $448 \mathrm{~A}-59-2(126)_{2}$ & 47 & 49.1 & 0.69 & 3.59 & 13.07 & 0.32 & 15.44 & 18.16 & 0.22 & 0.02 & $43 / 21 / 36$ \\
\hline $448 \mathrm{~A}-62-2(7)_{1}$ & 49 & 55.8 & 0.36 & 2.35 & 10.72 & 0.32 & 16.91 & 18.20 & 0.20 & 0.04 & $47 / 17 / 36$ \\
\hline $448 \mathrm{~A}-62-2(7)_{2}$ & 49 & 55.4 & 0.51 & 3.22 & 11.08 & 0.29 & 17.00 & 18,00 & 0.24 & 0.00 & $47 / 17 / 36$ \\
\hline \multicolumn{12}{|c|}{ Orthopyroxenes } \\
\hline $448-58-3(113)_{1}$ & 12 & - & - & - & 16.36 & - & 27.87 & 2.32 & - & - & $68 / 24 / 8$ \\
\hline $448-58-3(113)_{2}$ & 12 & 52.7 & - & 1.67 & 18.40 & - & 25.77 & 2.64 & 0.14 & 0.05 & $68 / 27 / 5$ \\
\hline $448-58-3(113)_{3}$ & 12 & - & - & - & 15.81 & - & 25.05 & 4.10 & 0.12 & 0.01 & $72 / 24 / 4$ \\
\hline $448-59-1(62)_{1}$ & 12 & 54.1 & 0.29 & 2.14 & 17.71 & - & 25.02 & 2.28 & 0.00 & 0.04 & $68 / 27 / 5$ \\
\hline $448-59-1(62) 2$ & 12 & 54.8 & 0.17 & 1.14 & 18.13 & 一 & 26.02 & 2.10 & 0.00 & 0.02 & $69 / 27 / 4$ \\
\hline $448 \mathrm{~A}-15-1(30)_{1}$ & 12 & 53.9 & 0.18 & 1.68 & 17.90 & - & 24.26 & 2.22 & 0.00 & 0.05 & $68 / 28 / 4$ \\
\hline 448 A- $15-1(30)_{2}$ & 12 & - & - & - & 17.53 & - & 27.31 & 2.04 & 0.00 & 0.05 & $71 / 25 / 4$ \\
\hline $448 \mathrm{~A}-51-1(85)_{1}$ & 37 & 51.7 & 0.36 & 1.86 & 21.38 & 0.55 & 18.93 & 4.88 & 0.16 & 0.06 & $55 / 35 / 10$ \\
\hline $448 \mathrm{~A}-51-1(85)_{2}$ & 37 & 52.6 & 0.28 & 1.70 & 20.25 & 0.60 & 20.33 & 5.02 & 0.10 & 0.08 & $58 / 32 / 10$ \\
\hline \multicolumn{12}{|c|}{ Olivine } \\
\hline & & & & & & & & & & & $\mathrm{Fo}^{\mathrm{c}}$ \\
\hline $448-37-1(52)$ & 6 & 36.59 & 0.00 & 0.00 & 32.65 & - & 30.76 & - & - & - & 63 \\
\hline \multicolumn{12}{|c|}{ Opaques } \\
\hline $448-50-3(80) \mathrm{MT}^{\mathrm{a}}$ & 9 & 0.88 & 4.66 & 0.74 & 81.53 & - & 0.39 & - & - & - & \\
\hline $448-50-3(80)_{\mathrm{IL}^{\mathrm{a}}}$ & 9 & 0.00 & 18.71 & 1.28 & 77.46 & - & 0.33 & - & - & - & \\
\hline $448 \mathrm{~A}-41-4(16)_{\mathrm{IL}}$ & 27 & 2.09 & 15.43 & 3.93 & 75.60 & 0.42 & 0.34 & 0.16 & 0.18 & 0.08 & \\
\hline $448 \mathrm{~A}-50-1(99)_{\mathrm{IL} 1}$ & 35 & 3.56 & 14.98 & 3.21 & 79.13 & 0.37 & 0.17 & 0.10 & 0.03 & 0.00 & \\
\hline $488 \mathrm{~A}-50-1(99)_{\mathrm{IL} 2}$ & 35 & 1.07 & 15.09 & 3.15 & 78.38 & 0.39 & 0.22 & 0.08 & 0.00 & 0.04 & \\
\hline $448 \mathrm{~A}-51-1(85)_{\mathrm{M}} \mathrm{T}$ & 37 & 0.13 & 9.13 & 4.43 & 80.21 & 0.30 & 2.95 & 0.20 & 0.00 & 0.02 & \\
\hline $448 \mathrm{~A}-56-1(31)_{\mathrm{IL}}$ & 41 & 2.49 & 13.63 & 3.00 & 78.80 & 0.33 & 0.49 & 0.13 & 0.01 & 0.05 & \\
\hline $448 \mathrm{~A}-59-2(126)_{\mathrm{ILI}}$ & 47 & 0.42 & 12.51 & 3.82 & 77.58 & 0.35 & 0.99 & 0.36 & 0.05 & 0.59 & \\
\hline $448 \mathrm{~A}-59-2(126)_{\mathrm{IL} 2}$ & 47 & 0.17 & 15.50 & 3.20 & 78.39 & 0.42 & 0.80 & 0.10 & 0.01 & 0.03 & \\
\hline $448 \mathrm{~A}-62-2(7)_{\mathrm{IL}}$ & 49 & 0.24 & 18.30 & 3.20 & 77.86 & 0.46 & 0.29 & 0.38 & 0.21 & 0.01 & \\
\hline $448-50-3(80)_{1}$ & 9 & 52.4 & 0.04 & 0.44 & 14.08 & 一 & 11.76 & 21.85 & 0.16 & 0.06 & \\
\hline $448-50-3(80)_{2}$ & 9 & 50.9 & 0.21 & 1.82 & 13.44 & - & 12.40 & 18.97 & 0.30 & 0.15 & \\
\hline \multicolumn{12}{|c|}{ Plagioclase } \\
\hline & & & & & & & & & & & $\mathrm{An}^{\mathrm{d}}$ \\
\hline $448-37-1(52)_{A}$ & 6 & 47.8 & 0.00 & 28.11 & - & - & - & 15.15 & 2.79 & 0.07 & 75 \\
\hline $448-37-1(52)_{\mathrm{H}}$ & 6 & 46.3 & 0.00 & 29.74 & - & - & - & 16.35 & 2.25 & 0.00 & 80 \\
\hline $448-37-1(52)_{\mathrm{L}}$ & 6 & 48.0 & 0.00 & 23.42 & - & - & - & 14.12 & 3.13 & 0.08 & 71 \\
\hline $448-39-1(75)_{\mathrm{A} 1}$ & 6 & 46.8 & 0.00 & 30.48 & - & - & - & 14.85 & 3.06 & 0.08 & 72 \\
\hline $448-39-1(75)_{\mathrm{HI}}$ & 6 & 46.4 & 0.00 & 31.11 & - & - & - & 15.26 & 2.74 & 0.09 & 75 \\
\hline $448-39-1(75)_{\mathrm{A} 2}$ & 6 & 48.6 & 0.00 & 29.07 & - & - & - & 13.66 & 3.48 & 0.08 & 68 \\
\hline $448-39-1(75)$ L2 & 6 & 48.8 & 0.00 & 28.64 & - & - & - & 13.62 & 3.65 & 0.03 & 67 \\
\hline $448-39-1(75)_{\mathrm{A} 3}$ & 6 & - & 0.00 & - & - & - & - & 12.27 & 3.83 & 0.08 & 64 \\
\hline $448-47-2(22)_{\mathrm{A}}$ & 8 & 48.2 & 0.00 & 28.13 & - & - & - & 12.01 & 3.71 & 0.16 & 63 \\
\hline $448-50-3(80)_{A}$ & 9 & 53.1 & 0.00 & 26.82 & - & - & - & 9.31 & 5.91 & 0.15 & 46 \\
\hline $448-50-3(80)_{\mathrm{H}}$ & 9 & 51.5 & 0.00 & 27.07 & - & - & - & 10.98 & 5.06 & 0.12 & 54 \\
\hline $448-50-3(80)_{L}$ & 9 & 57.1 & 0.00 & 23.70 & - & - & - & 5.62 & 7.73 & 0.71 & 27 \\
\hline $448-53-1(2)_{A}$ & 10 & 43.4 & 0.00 & 33.77 & - & - & - & 13.30 & 3.40 & 0.07 & 68 \\
\hline $448-58-3(113)_{\mathrm{A} 1}$ & 12 & 48.7 & 0.00 & 32.80 & - & - & - & 16.35 & 2.09 & 0.05 & 81 \\
\hline $448-58-3(113)_{\mathrm{H} 2}$ & 12 & 47.6 & 0.00 & 33.25 & - & - & - & 16.79 & 1.69 & 0.04 & 84 \\
\hline 448A-58-3 (113)L2 & 12 & 49.3 & 0.00 & 32.21 & - & - & - & 15.96 & 2.54 & 0.07 & 77 \\
\hline $448-59-1(62)_{\mathrm{A} 1}$ & 12 & 46.0 & 0.00 & 32.93 & - & - & - & 16.23 & 1.97 & 0.07 & 82 \\
\hline $448-59-1(62)_{A 2}$ & 12 & 45.9 & 0.00 & 32.79 & - & - & - & 16.13 & 2.09 & 0.07 & 81 \\
\hline $448-59-3(51)_{\mathrm{A}}$ & 12 & 45.2 & 0.00 & 32.20 & - & - & - & 16.32 & 2.13 & 0.13 & 80 \\
\hline
\end{tabular}


Table 3. (Continued).

\begin{tabular}{|c|c|c|c|c|c|c|c|c|c|c|c|}
\hline Sample $^{\mathrm{a}}$ & Unit & $\mathrm{SiO}_{2}$ & $\mathrm{TiO}_{2}$ & $\mathrm{Al}_{2} \mathrm{O}_{3}$ & $\mathrm{FeO}^{*}$ & $\mathrm{MnO}$ & $\mathrm{MgO}$ & $\mathrm{CaO}$ & $\mathrm{Na}_{2} \mathrm{O}$ & $\mathrm{K}_{2} \mathrm{O}$ & $\mathrm{En} / \mathrm{Fs}_{s} / \mathrm{Wo}_{0}^{\mathrm{b}}$ \\
\hline \multicolumn{12}{|c|}{ Clinopyroxenes } \\
\hline $448 \mathrm{~A}-15-1(30)_{\mathrm{L} 1}$ & 12 & 47.0 & 0.00 & 33.50 & - & - & - & 12.50 & 3.62 & 0.17 & 67 \\
\hline $448 \mathrm{~A}-15-1(30) \mathrm{Al}$ & 12 & 47.8 & 0.00 & 33.63 & - & - & - & 15.76 & 2.49 & 0.07 & 77 \\
\hline $448 \mathrm{~A}-15-1(30)_{\mathrm{H} 1}$ & 12 & 42.5 & 0.00 & 33.02 & - & - & - & 17.03 & 1.67 & 0.04 & 85 \\
\hline $448 \mathrm{~A}-15-1{ }^{(30)} \mathrm{H} 2$ & 12 & 46.5 & 0.00 & 34.33 & - & - & - & 16.88 & 1.79 & 0.09 & 83 \\
\hline $448 \mathrm{~A}-15-1(30)_{\mathrm{A} 2}$ & 12 & 47.8 & 0.00 & 33.59 & - & - & - & 15.35 & 2.35 & 0.10 & 78 \\
\hline $448 \mathrm{~A}-15-1(30)_{\mathrm{L} 2}$ & 12 & 47.0 & 0.00 & 33.44 & - & - & - & 11.75 & 3.40 & 0.26 & 64 \\
\hline $448 \mathrm{~A}-18-1(112)_{\mathrm{A} 1}$ & 14 & 43.3 & 0.00 & 33.70 & - & - & - & 16.34 & 2.17 & 0.05 & 80 \\
\hline $448 \mathrm{~A}-18-1(112) \mathrm{A} 2$ & 14 & 46.2 & 0.00 & 33.10 & - & - & - & 16.39 & 1.98 & 0.04 & 82 \\
\hline $448 \mathrm{~A}-18-1(112) \mathrm{A} 3$ & 14 & 44.0 & 0.00 & 34.70 & - & - & - & 17.39 & 1.45 & 0.05 & 87 \\
\hline $448 \mathrm{~A}-41-4(16) \mathrm{L} 1$ & 27 & 51.9 & 0.00 & 30.08 & - & - & - & 14.56 & 3.58 & 0.13 & 69 \\
\hline $448 \mathrm{~A}-41-4(16)_{\mathrm{A} 1}$ & 27 & 48.9 & 0.00 & 32.14 & 0.83 & 0.01 & 0.15 & 16.80 & 2.48 & 0.09 & 78 \\
\hline $448 \mathrm{~A}-41-4(16) \mathrm{H} 1$ & 27 & - & - & - & - & - & - & 16.13 & 2.13 & 0.08 & 80 \\
\hline $448 \mathrm{~A}-41-4(16)_{\mathrm{A} 2}$ & 27 & 47.7 & 0.00 & 32.91 & 0.77 & 0.04 & 0.13 & 17.15 & 1.99 & 0.08 & 82 \\
\hline $448 \mathrm{~A}-41-4(16) \mathrm{L} 2$ & 27 & 51.6 & 0.00 & 30.60 & - & - & - & 14.51 & 3.86 & 0.16 & 67 \\
\hline $448 \mathrm{~A}-41-4(16) \mathrm{H} 2$ & 27 & 48.1 & 0.00 & 33.52 & - & - & - & 18.18 & 1.49 & 0.16 & 86 \\
\hline $448 \mathrm{~A}-50-1\left({ }^{(99)} \mathrm{A} 1\right.$ & 35 & 49.9 & 0.00 & 32.97 & 1.01 & 0.01 & 0.15 & 16.21 & 2.51 & 0.05 & 78 \\
\hline $448 \mathrm{~A}-50-1(99)_{\mathrm{H} 1}$ & 35 & 51.9 & 0.00 & 31.80 & - & - & - & 17.75 & 2.13 & 0.04 & 82 \\
\hline $448 \mathrm{~A}-50-1(99)_{\mathrm{L} 1}$ & 35 & 55.7 & 0.00 & 29.21 & - & - & - & 10.09 & 4.87 & 0.30 & 52 \\
\hline $448 \mathrm{~A}-50-1(99)_{\mathrm{L} 2}$ & 35 & 51.2 & 0.00 & 31.54 & 0.88 & 0.00 & 0.16 & 14.51 & 3.84 & 0.10 & 67 \\
\hline $448 \mathrm{~A}-50-1(99)_{\mathrm{H} 2}$ & 35 & 49.0 & 0.00 & 33.64 & - & - & - & 17.47 & 2.00 & 0.06 & 83 \\
\hline $448 \mathrm{~A}-51-1(85)_{\mathrm{A} 1}$ & 37 & 52.8 & 0.00 & 28.52 & 1.29 & 0.02 & 0.20 & 13.37 & 4.13 & 0.10 & 64 \\
\hline $448 \mathrm{~A}-51-1(85)_{\mathrm{A} 2}$ & 37 & 47.4 & 0.00 & 32.19 & 0.90 & 0.01 & 0.11 & 16.56 & 1.94 & 0.06 & 82 \\
\hline $448 \mathrm{~A}-56-1(31)_{\mathrm{A} 1}$ & 41 & 44.8 & 0.00 & 34.40 & 0.65 & 0.01 & 0.12 & 19.47 & 0.84 & 0.04 & 93 \\
\hline $448 \mathrm{~A}-56-1(31) \mathrm{A} 2$ & 41 & 44.3 & 0.00 & 34.64 & 0.71 & 0.00 & 0.16 & 19.44 & 0.79 & 0.06 & 93 \\
\hline $448 \mathrm{~A}-59-2(126)_{\mathrm{A} 1}$ & 47 & 48.2 & 0.00 & 31.42 & 0.82 & 0.02 & 0.14 & 16.85 & 2.38 & 0.06 & 79 \\
\hline $448 \mathrm{~A}-59-2(126) \mathrm{L} 1$ & 47 & 51.6 & 0.00 & 29.64 & - & - & - & 15.28 & 2.77 & 0.05 & 71 \\
\hline $448 \mathrm{~A}-59-2(126) \mathrm{H} 1$ & 47 & 46.5 & 0.00 & 32.56 & - & - & - & 17.81 & 4.38 & 0.09 & 83 \\
\hline $448 \mathrm{~A}-59-2(126)_{\mathrm{A} 2}$ & 47 & 47.4 & 0.00 & 31.65 & - & - & - & 15.91 & 1.90 & 0.02 & 76 \\
\hline $448 \mathrm{~A}-59-2(126) \mathrm{L} 1$ & 47 & 49.9 & 0.00 & 29.63 & - & - & - & 13.21 & 4.13 & 0.10 & 62 \\
\hline $448 \mathrm{~A}-59-2(126) \mathrm{H} 2$ & 47 & 46.4 & 0.00 & 32.74 & - & - & - & 17.43 & 1.94 & 0.06 & 83 \\
\hline $448 \mathrm{~A}-62-2(7) \mathrm{H} 1$ & 49 & 45.4 & 0.00 & 33.47 & 0.78 & 0.00 & 0.16 & 17.74 & 1.28 & 0.02 & 88 \\
\hline $448 \mathrm{~A}-62-2(7)_{\mathrm{L} 1}$ & 49 & 48.7 & 0.00 & 32.01 & - & - & - & 13.50 & 3.96 & 0.07 & 65 \\
\hline $448 \mathrm{~A}-62-2(7)_{\mathrm{A} 1}$ & 49 & 46.7 & 0.00 & 33.08 & - & - & - & 16.17 & 1.93 & 0.09 & 82 \\
\hline $448 \mathrm{~A}-62-2(7) \mathrm{A} 2$ & 49 & 54.2 & 0.00 & 34.45 & 0.79 & 0.01 & 0.13 & 15.22 & 2.87 & 0.09 & 74 \\
\hline $448 \mathrm{~A}-62-2(7) \mathrm{L} 2$ & 49 & 57.3 & 0.00 & 32.04 & - & - & - & 12.69 & 4.44 & 0.14 & 61 \\
\hline $448 \mathrm{~A}-62-2(7)_{\mathrm{H} 2}$ & 49 & 52.7 & 0.00 & 35.52 & - & - & - & 16.50 & 2.03 & 0.07 & 81 \\
\hline \multicolumn{12}{|c|}{ Zeolites } \\
\hline $448-37-1(52)$ & 6 & 59.2 & 0.00 & 18.66 & - & - & - & 0.02 & 4.64 & 7.95 & \\
\hline $448-58-3$ (113) & 12 & 59.4 & 0.00 & 18.71 & - & - & - & 0.04 & 5.53 & 7.58 & \\
\hline $448 \mathrm{~A}-16-2(71)$ & 12 & 59.3 & 0.00 & 18.67 & - & - & - & 0.07 & 4.83 & 7.61 & \\
\hline $448 \mathrm{~A}-18-1(112)$ & 14 & 59.4 & 0.00 & 18.73 & - & - & - & 0.05 & 5.90 & 7.59 & \\
\hline
\end{tabular}

and trace-element criteria are of value, particularly in light of the severe alteration of Site 448 ridge rocks. Table 5 is a brief summary of discrimination criteria. There is no question that the flows, dikes, and sills are all arc tholeiites. The high $\mathrm{Fe} / \mathrm{Mg}$, low $\mathrm{Al}_{2} \mathrm{O}_{3}$, intermediate $\mathrm{K}_{2} \mathrm{O}$, intermediate $\mathrm{Ba}$, and low $\mathrm{Sr}, \mathrm{Ni}, \mathrm{Cr}$, and light (L) REE/heavy (H) REE values are the principal distinguishing features of arc tholeiites. No sample falls outside the trend except one clast-Sample 448A-21-1 (56) - which has a normalized $\mathrm{La} / \mathrm{Lu}$ ratio of 1.15 and an average enrichment relative to carbonaceous chondrites of about 30 (Fig. 6). The hornblende diorite that is also somewhat enriched in REE has a normalized $\mathrm{La} / \mathrm{Lu}$ ratio of 0.84 , clearly of arc-tholeiitic character. The slight LREE-enrichment that is related to overall REE-enrichment in these two rocks is probably a result of crystal fractionation rather than calc-alkalic origins, because the heavy REE are also enriched (Jakes and Gill, 1970). Migdisov (this volume) has studied the tuffs in Units 4 and 5 of Hole 448 and reports major-element evidence that is suggestive of calc-alkalic volcanism. He found samples with the following average chemical character: $\mathrm{Al}_{2} \mathrm{O}_{3}=13.7 \%, \mathrm{Ba}=49 \mathrm{ppm}$, and $\mathrm{Sr}=169$ $\mathrm{ppm}$. These values are impressively close to the average values for all the flows at Site $448\left(\mathrm{Al}_{2} \mathrm{O}_{3}=14.0 \%\right.$, $\mathrm{Ba}$ $=49 \mathrm{ppm}, \mathrm{Sr}=169 \mathrm{ppm}$ ); therefore these tuffs can be considered to be arc-tholeiitic series differentiates. No evidence exists at Site 448 that volcanism on the PalauKyushu Ridge ever reached the calc-alkalic stage before volcanism began to wane in the middle late Oligocene. However, the data reported by the International Working Group on the IGCP Project "Ophiolites" (1977) indicate that the basaltic andesites do appear to have calcalkalic affinities. For example, one sample has $19.4 \%$ $\mathrm{Al}_{2} \mathrm{O}_{3}, 70 \mathrm{ppm} \mathrm{Ba}$, and $340 \mathrm{ppm} \mathrm{Sr}$. Although the $\mathrm{Ba}$ value is somewhat low for typical calc-alkalic rocks, the $\mathrm{Al}_{2} \mathrm{O}_{3}$ and $\mathrm{Sr}$ values do fit. It may be tentatively concluded that, at least locally, some calc-alkalic volcanism occurred on the Palau-Kyushu Ridge, even though conclusive evidence is wanting. In contrast, the XRF data 
Table 4. Rare-earth element and other trace-element chemistry of whole rocks from Site $448{ }^{\mathrm{a}}$

\begin{tabular}{|c|c|c|c|c|c|c|c|c|c|c|c|c|c|}
\hline Sample ${ }^{b}$ & Unit & $\mathrm{La}$ & $\mathrm{Ce}$ & $\mathrm{Sm}$ & $\mathrm{Eu}$ & $\mathrm{Tb}$ & $\mathrm{Yb}$ & $\mathrm{Lu}$ & Co & $\mathrm{Cr}$ & $\mathrm{FeO}^{*}$ & Sc & $\mathrm{Hf}$ \\
\hline $448-39-1(75)$ & 6 & 4.5 & 12.1 & 2.02 & 0.97 & 0.38 & 2.5 & 0.72 & 42 & 35 & 14.69 & 43 & 1.05 \\
\hline $448-48-1$ (119) & 8 & 10.2 & 12.1 & 3.17 & 1.37 & 0.60 & 3.7 & 0.70 & 34 & 10 & 15.29 & 44 & 1.09 \\
\hline $448-50-3(80)$ & 9 & 17.9 & 18.4 & 4.43 & 1.38 & 0.17 & 4.2 & 0.84 & 34 & 6 & 11.20 & 35 & 2.26 \\
\hline $448-53-1(2)$ & 10 & 7.8 & 13.5 & 2.61 & 1.16 & 0.44 & 2.7 & 0.37 & 44 & 23 & 12.58 & 43 & 1.08 \\
\hline $448-55-1(110)$ & 11 & 8.2 & 11.5 & 2.56 & 0.94 & 0.36 & 1.6 & 0.25 & 20 & 10 & 6.49 & 34 & 0.80 \\
\hline $448 \mathrm{~A}-15-1(30)$ & 12 & 10.4 & 14.8 & 3.02 & 1.13 & 0.45 & 3.1 & 0.45 & 35 & 25 & 11.09 & 38 & 1.57 \\
\hline $448 \mathrm{~A}-21-1(56)$ & 15 & 32.8 & 25.1 & 8.15 & 2.70 & 130 & 5.2 & 0.98 & 33 & 13 & 10.28 & 45 & 1.68 \\
\hline $448 \mathrm{~A}-26-2(32)$ & 16 & 6.6 & 10.6 & 2.18 & 1.02 & 0.38 & 2.3 & 0.38 & 37 & 30 & 11.82 & 42 & 0.64 \\
\hline 448A-28-1 (96) & 18 & 4.8 & 10.4 & 1.48 & 0.83 & 0.27 & 1.4 & 0.35 & 37 & 49 & 11.25 & 43 & 0.65 \\
\hline $448 \mathrm{~A}-33-1$ (134) & 20 & 7.6 & 10.7 & 2.58 & 1.13 & 0.43 & 3.2 & 0.48 & 115 & 8 & 14.37 & 44 & 0.81 \\
\hline $448 \mathrm{~A}-36-5(53)$ & 22 & 6.0 & 13.0 & 1.90 & 0.80 & 0.35 & 1.9 & 0.32 & 46 & 44.7 & 10.88 & 38 & 0.88 \\
\hline $448 \mathrm{~A}-37-2(141)$ & 23 & 6.3 & 9.8 & 2.58 & 1.12 & 0.55 & 3.3 & 0.51 & 49 & 70 & 12.48 & 43 & 0.97 \\
\hline $448 \mathrm{~A}-40-1(33)$ & 24 & 10.6 & 11.9 & 3.08 & 1.15 & 0.49 & 3.0 & 0.55 & 42 & 12 & 13.34 & 39 & 1.06 \\
\hline $448 \mathrm{~A}-41-1(90)$ & 26 & 6.0 & 7.8 & 2.21 & 1.02 & 0.42 & 2.6 & 0.44 & 33 & 25 & 11.41 & 40 & 0.54 \\
\hline $448 \mathrm{~A}-41-2(12)_{1}$ & 27 & 4.7 & 9.8 & 1.82 & 0.88 & 0.28 & 1.8 & 0.38 & 46 & 29 & 11.87 & 41 & 0.60 \\
\hline $448 \mathrm{~A}-45-1(43)$ & 31 & 5.5 & 11.4 & 2.03 & 1.08 & 0.37 & 2.4 & 0.46 & 37 & 38 & 11.45 & 43 & 0.65 \\
\hline $448 \mathrm{~A}-47-3(138)_{1}$ & 33 & 8.1 & 12.5 & 1.03 & 1.12 & 0.52 & 3.2 & 0.49 & 37 & 12 & 12.49 & 41 & 0.96 \\
\hline $448 \mathrm{~A}-50-1(95)$ & 35 & 6.1 & 11.1 & 2.54 & 1.09 & 0.43 & 2.9 & 0.51 & 41 & 26 & 13.41 & 42 & 0.89 \\
\hline $448 \mathrm{~A}-51-3(135)$ & 37 & 7.3 & 11.1 & 2.72 & 1.12 & 0.46 & 3.0 & 0.53 & 33 & 63 & 11.50 & 26 & 0.90 \\
\hline $448 \mathrm{~A}-52-2(128)$ & 39 & 7.8 & 11.4 & 2.50 & 1.03 & 0.47 & 2.7 & 0.43 & 32 & 26 & 13.10 & 37 & 0.64 \\
\hline $448 \mathrm{~A}-54-3(81)$ & 41 & 7.2 & 10.9 & 2.58 & 1.05 & 0.42 & 3.7 & 0.49 & 44 & 14 & 11.36 & 41 & 0.96 \\
\hline $448 \mathrm{~A}-59-2(126)$ & 47 & 5.9 & 8.6 & 2.51 & 1.09 & 0.51 & 2.9 & 0.50 & 49 & 36 & 11.62 & 43 & 0.71 \\
\hline $448 \mathrm{~A}-62-2(7)$ & 49 & 9.0 & 11.3 & 2.06 & 0.74 & 0.35 & 2.0 & 0.37 & 30 & 26 & 9.72 & 36 & 0.76 \\
\hline $448 \mathrm{~A}-65-2(125)$ & 51 & 6.0 & 9.8 & 2.27 & 0.87 & 0.41 & 2.9 & 0.45 & 31 & 21 & 11.41 & 42 & 0.71 \\
\hline
\end{tabular}

a All elements are reported as ppm except $\mathrm{Fe}$, which is per cent $\mathrm{FeO}$.

b Sample numbers refer to the hole-core-section and center of the sample interval (in parentheses). Subscript 1 refers to the area analyzed indicated in Table 2.

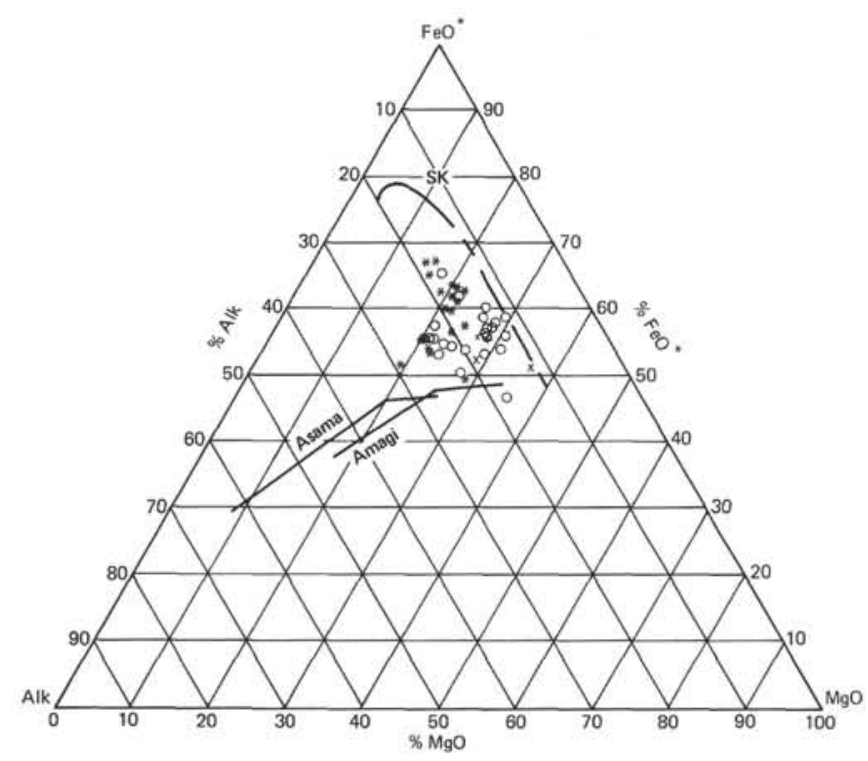

Figure 1. AFM plot $\left(\mathrm{Na}_{2} \mathrm{O}+\mathrm{K}_{2} \mathrm{O}, \mathrm{FeO} *, \mathrm{MgO}\right.$ wt. $\%$, where $\mathrm{FeO}^{*}$ is total $\mathrm{Fe}$ expressed as $\mathrm{FeO}$ ) of XRF data for Site 448. (Hole 448 samples of igneous units are shown by asterisks, Hole 448 clasts from volcaniclastic breccia units by pluses, Hole 448A samples of igneous units by dotted circles, and Hole 448A clasts from volcaniclastic breccia units by $X$ 'es. The trend lines for the tholeiitic Skaergaard [SK] intrusion and the calc-alkalic Asama and Amagi volcanoes are shown for comparison.)

from Site 451 on the West Mariana Ridge indicate a strong calc-alkalic character: $\mathrm{Ba}$ averages $185 \mathrm{ppm} ; \mathrm{Sr}$, $525 \mathrm{ppm} ; \mathrm{Al}_{2} \mathrm{O}_{3}, 18.2 \%$. Thus it appears that some evolutionary trends exist in the progression of arc activity from the Palau-Kyushu to West Mariana ridges.

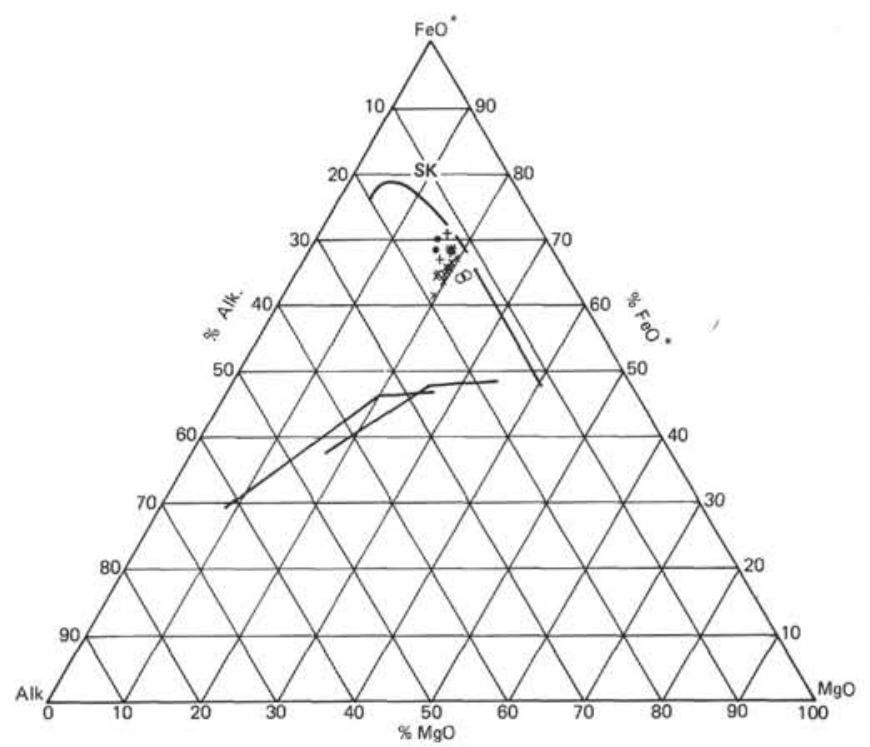

Figure 2. AFM plot of probe data for fresh glasses from Site 448. (The different symbols refer to volcanic Units 6 [indicated by ${ }^{*}$ ], $8[+]$, $10[\odot], 12$ and $14[\times]$, and $37[\bullet]$. Note that the grouping has a slight alkali-enrichment trend.)

The serious effect of pervasive alteration in Site 448 rocks is even more exaggerated by normative plots: when XRF data are plotted on the normative olivine-diopside-quartz diagram of Shibata (1976), variations occur ranging from highly quartz normative to nepheline normative along a silica control trend, with some scattering into the Mg-rich part of the olivine field (Fig. 7). The redistribution of silicon and enrichment of 


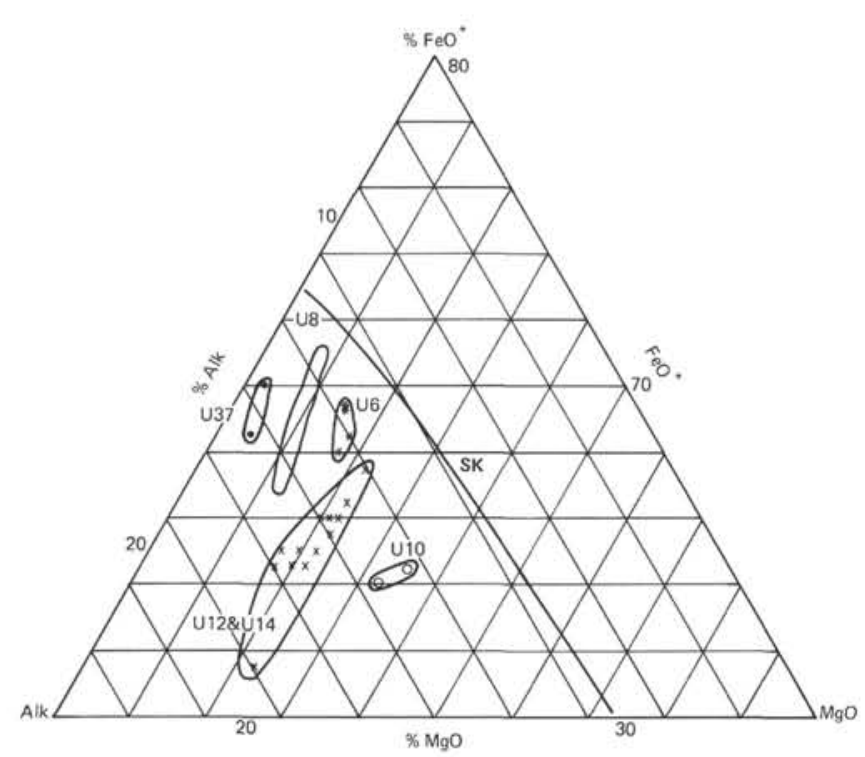

Figure 3. Expanded AFM plot of $5 \%$ to $25 \%$ alkalis, $60 \%$ to $80 \%$ $\mathrm{FeO}^{*}$, and $15 \%$ to $30 \% \mathrm{MgO}$ of data shown in Figure 2.

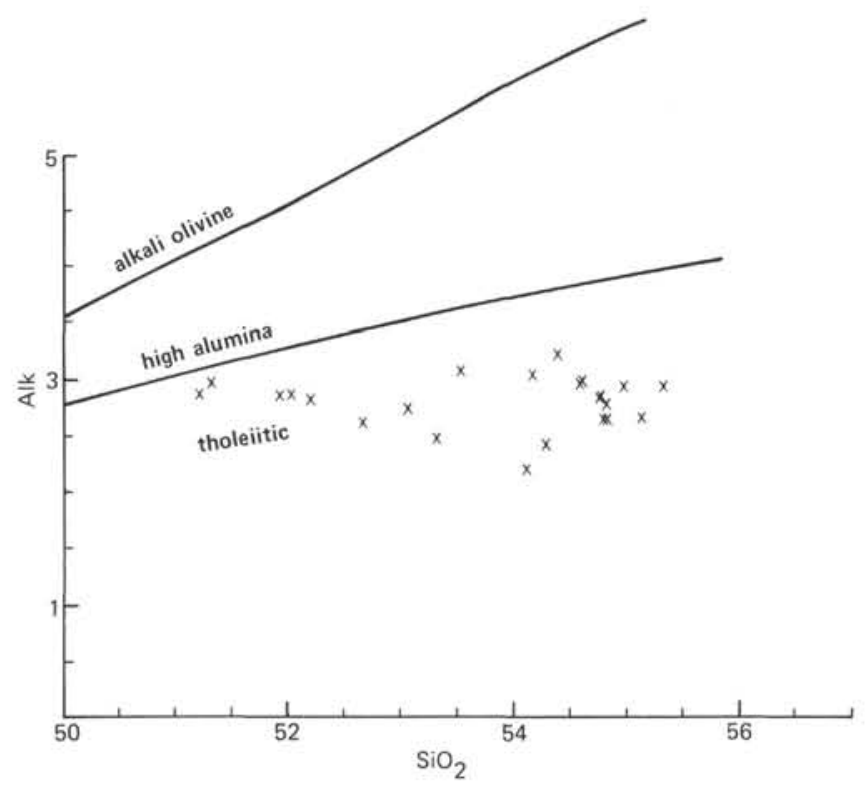

Figure 4. Plot $\mathrm{SiO}_{2}$ versus $\mathrm{Na}_{2} \mathrm{O}+\mathrm{K}_{2} \mathrm{O}$ (wt. \%) for fresh glass probe analyses from Site 448 . (Fields of alkali-olivine basalt, highalumina basalt, and tholeiitic basalt and differentiates are shown after Kuno [1965].)

magnesium in basalts during hydrothermal reaction with sea water (Hajash, 1975) is well established and probably is responsible for most of the variations observed. Obviously, this alteration makes normative diagrams petrologically useless. A signficant contrast occurs with the fresh glass analyses, which cluster tightly between the $1 \mathrm{~atm}$ (Fig. 8) and the natural oceanic-tholeiite two-pyroxene cotectic. Also, the position of the glasses about the two-pyroxene cotectic conforms with the petrographic observations of coexisting pyroxenes and rare olivine; apparently the liquid compositions have all left the olivine-clinopyroxene-ortho-

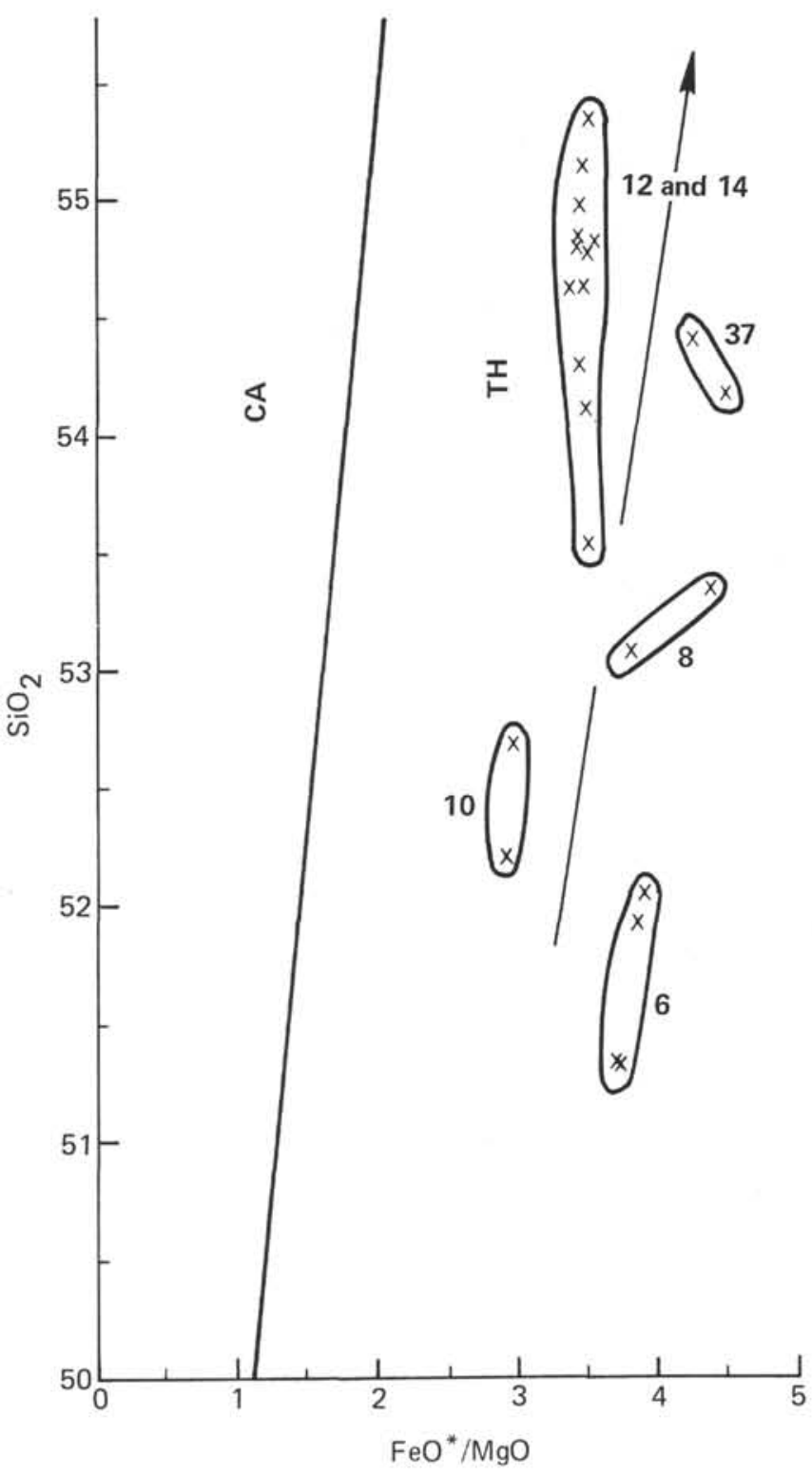

Figure 5. Plot of $\mathrm{FeO} * / \mathrm{MgO}$ versus $\mathrm{SiO}_{2}$ (wt. \%) for fresh glass probe analyses from Site 448. (The slope of the line separating calc-alkalic [CA] and tholeiitic [TH] differentiation trends is shown according to Miyashiro [1974].)

pyroxene reaction point and any olivine phenocrysts that remain must be relic and in disequilibrium.

Confirmation of a clinopyroxene-orthopyroxene control on liquid fractionation can be found in the behavior of $\mathrm{Co}$ and $\mathrm{Cr}$. Because the trend of the twopyroxene cotectic is toward the quartz apex, the degree of pyroxene fractionation can be estimated. Both $\mathrm{Cr}$ and $\mathrm{Co}$ are preferentially removed by pyroxenes, which depletes residual liquids in these trace metals; this behavior is demonstrated in Figure 9, which shows decreasing $\mathrm{Cr}$ and $\mathrm{Co}$ contents and an increasing percentage of normative quartz in the olivine-diopsidequartz diagram.

Microprobe studies of the pyroxenes in the glasses and holocrystalline rocks indicate that the compositions of coexisting ortho- and clinopyroxenes (tie lines) follow 
Table 5. Comparison of selected trace- and majorelement criteria for discrimination among tholeiitic mid-ocean-ridge basalts, arc-tholeiite series differentiates, and calc-alkalic series differentiates.

\begin{tabular}{lcccc}
\hline Elements $^{\mathrm{a}}$ & MORB $^{\mathrm{b}}$ & $\mathrm{CA}^{\mathrm{c}}$ & AT $^{\mathrm{d}}$ & Site 448 \\
\hline $\mathrm{Fe} / \mathrm{Mg}$ & 1.11 & 1.4 & $<2.0$ & 4.6 \\
$\mathrm{Al}_{2} \mathrm{O}_{3} \%$ & 16.6 & 17.7 & 15.6 & 13.8 \\
$\mathrm{~K}_{2} \mathrm{O} \%$ & 0.07 & 1.25 & 0.43 & 0.45 \\
$\mathrm{Ba} \mathrm{ppm}$ & 10 & 300 & 50 & 49 \\
$\mathrm{Sr} \mathrm{ppm}$ & 110 & 400 & 200 & 169 \\
$\mathrm{Ni} \mathrm{ppm}$ & 150 & 20 & 20 & 14 \\
$\mathrm{Cr} \mathrm{ppm}$ & 400 & 50 & 20 & 25 \\
LREE/HREE & $<1$ & $\sim 10$ & $\leq 1$ & $<1$ \\
\hline
\end{tabular}

a The $\mathrm{Fe} / \mathrm{Mg}$ and $\mathrm{K}$ data for Site 448 are from probe analyses; $\mathrm{Al}$ and trace-element material from XRF data; and REE information from INAA data.

$\mathrm{b}$ MORB = mid-ocean-ridge basalts, from Johnson (1979), Bass et al. (1973), and Carmichael et al. 1974).

${ }^{\mathrm{c}} \mathrm{CA}=$ calc-alkalic series differentiates, from Jakeš and Gill (1970) and Taylor (1969).

$\mathrm{d}_{\mathrm{AT}}=$ arc-tholeiite series differentiates, from Jakeš and Gill (1970).

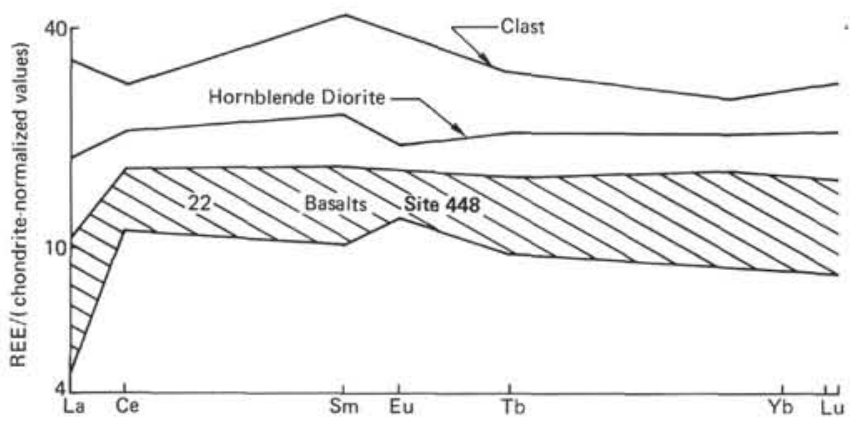

Figure 6. Rare-earth elements (REE) normalized to carbonaceous chondrites versus atomic sequence. (The basalt data [22] fall into a narrow range indistinguishable from those reported by Jakeš and Gill [1970]. The hornblende diorite clast-Sample 448-50-3, [80] - and another clast-Sample 448A-21-1 [56] - have higher overall REE contents and very small LREE enrichment that are the result of crystal fractionation rather than the influence of a calc-alkalic magmatic source.)

well recognized trends. Figure 10 shows the actual compositions determined in glasses: orthopyroxenes with about 2 to 2.5 mole percent wollastonite exist between about 78 and 71 mole percent enstatite, whereas pigeonitic pyroxenes with 10 mole percent wollastonite are found below about 68 mole percent enstatite. With the exception of one sample, a distict immiscibility field exists between low-Ca pyroxenes and augite that contains at least 26 mole percent wollastonite. The one olivine is shown with a tie line to coexisting augite. Figure 11 generalizes these fractionation trends and shows the much more limited field of clinopyroxene in holocrystalline rocks with a much larger immiscibility field, as expected.

The compositions of plagioclases are shown in Figures 12 and 13; the degrees of zoning in the units are indicated by brackets. Note that the plagioclases in glasses (Fig. 12) are considerably more sodic than are

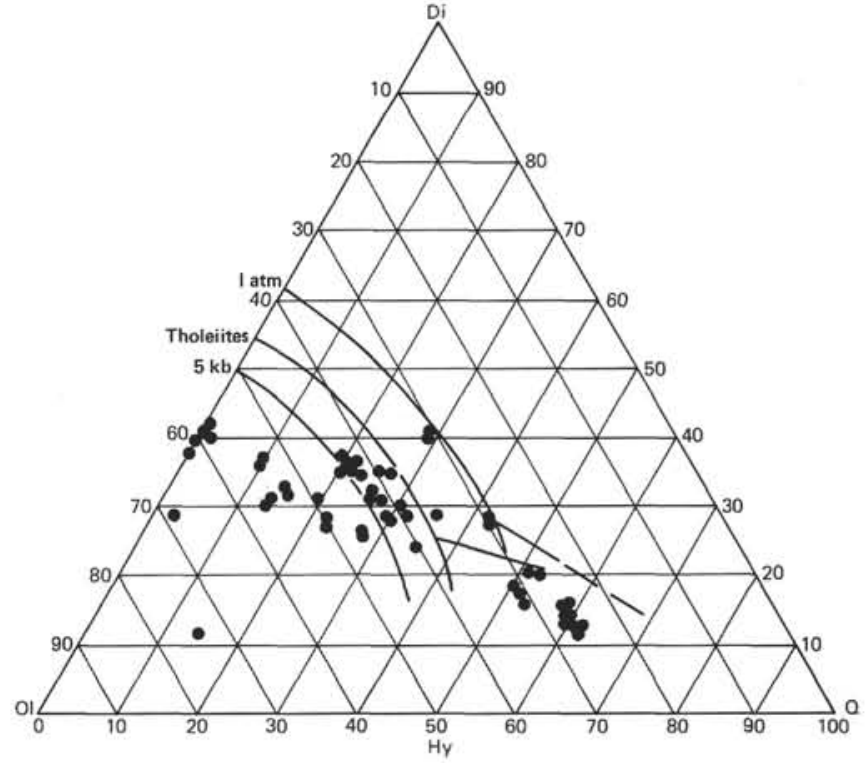

Figure 7. Normative olivine-diopside-quartz diagram after Shibata (1976), showing the compositions of samples analyzed by XRF. (The $1 \mathrm{~atm}, 5 \mathrm{~kb}$, and the natural oceanic tholeiitic cotectics for olivine-diopside, hypersthene-diopside, and olivine-hypersthene are indicated. The three samples on the olivine-diopside join actually fall in the nepheline normative field.)

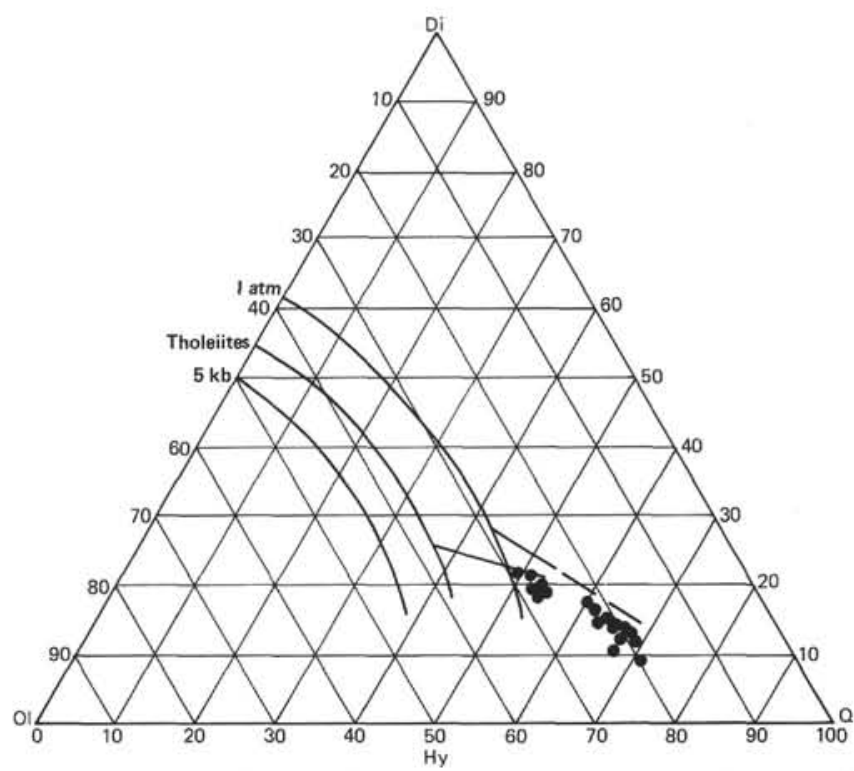

Figure 8. Normative olivine-diopside-quartz diagram after Shibata (1976), showing the compositions of fresh glasses by probe analysis (same diagram as Fig. 7).

those holocrystalline rocks (Fig. 13); the highly fractionated diorite is included with the glassy rocks. A distinct and progressive increase in solid solution of $\mathrm{K}$-feldspar in the plagioclase, with increasing $\mathrm{Na}$ feldspar content, is apparent in Figure 12.

In all the whole-rock and microprobe chemical studies of individual phases, no obvious trends or patterns are present that can be correlated with stratigraphic position. The petrology of the Palau-Kyushu 


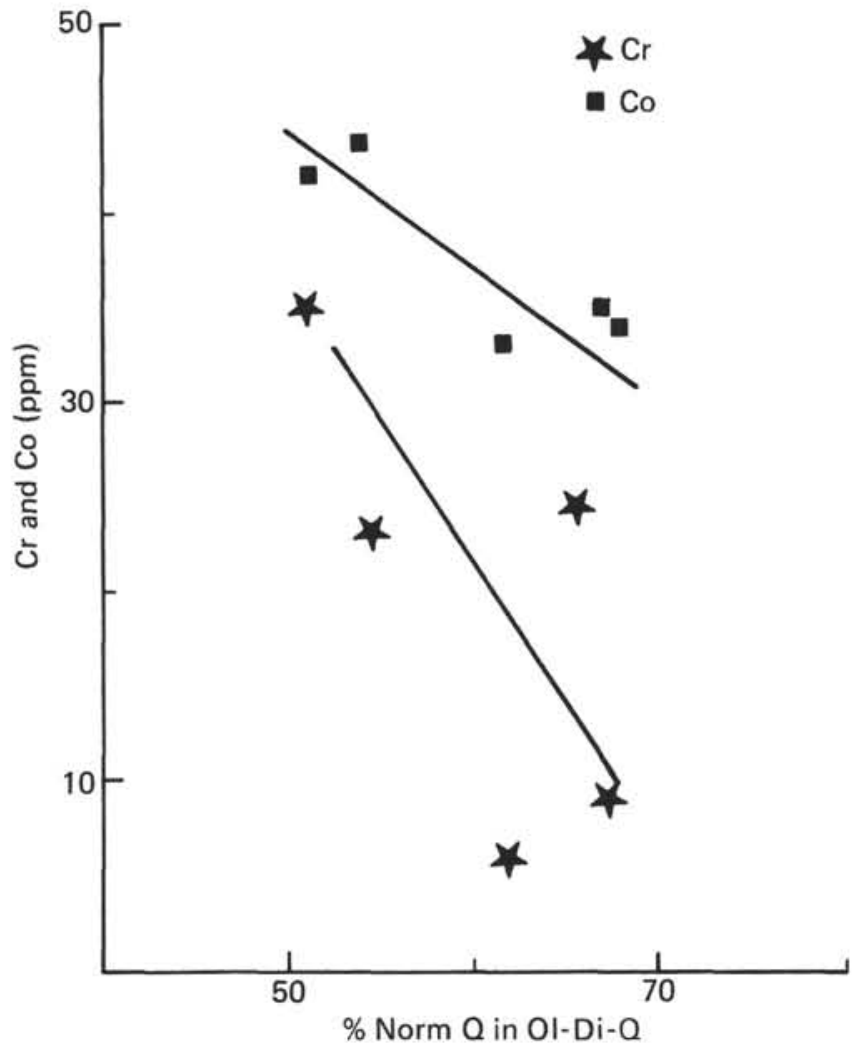

Figure 9. Concentration of $\mathrm{Cr}$ and $\mathrm{Co}$ versus the percentage of normative quartz in the olivine-diopside-quartz diagram. (The inverse trace-metal to normative-quartz relationship is interpreted as the result of progressive pyroxene-crystal fractionation. The Co and $\mathrm{Cr}$ data were measured by INAA, and the normative-quartz content of the glasses was calculated from probe data.)

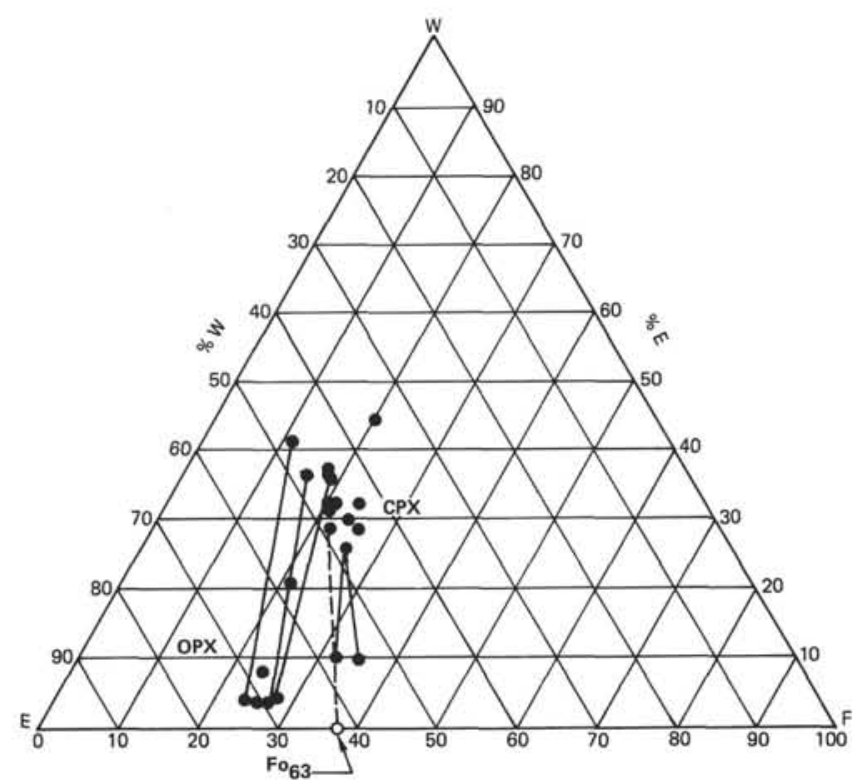

Figure 10. Composition of pyroxenes analyzed by microprobe in glasses plotted on the normative pyroxene diagram. (Solid lines are pyroxene tie lines, and the dashed line is the augite-olivine tie line, if the enstatite-ferrosilite join represents the forsterite-fayalite join.)

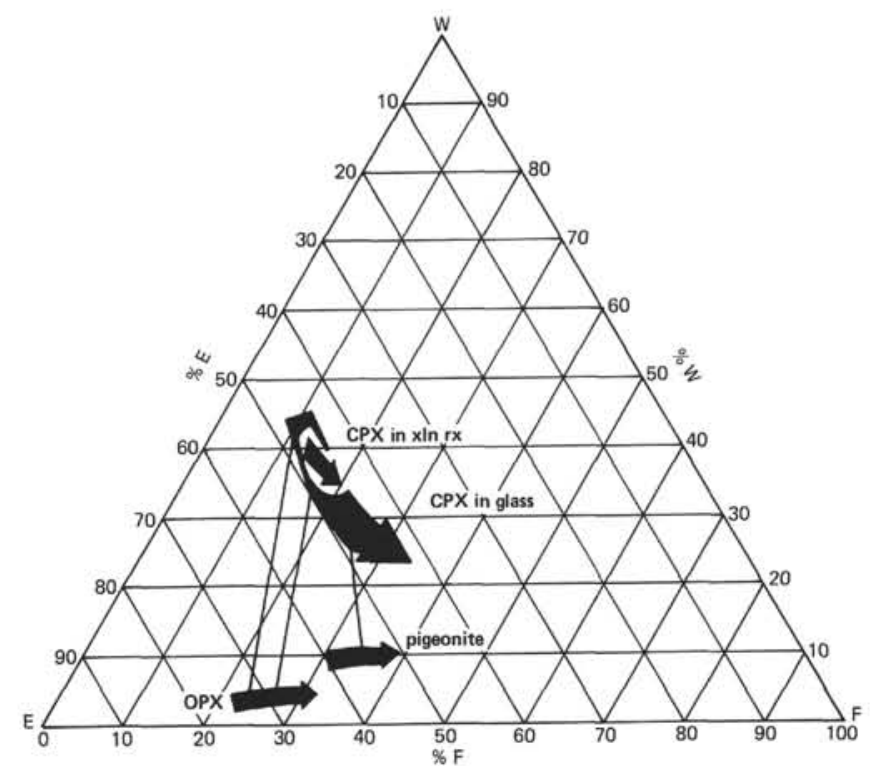

Figure 11. Generalized fractionation trends of pyroxenes in glass and holocrystalline rocks (xln rx) (same diagram as Fig. 10).

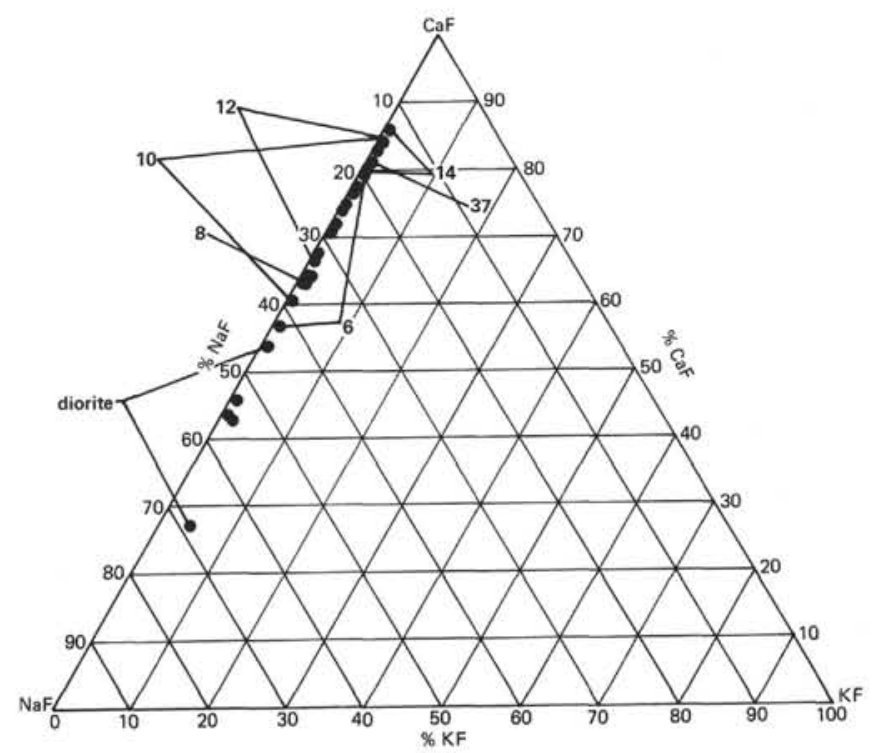

Figure 12. Normative ternary feldspar diagram, showing compositions of plagioclase from glasses. (The brackets show the degree of zoning of plagioclases for the indicated units. Although holocrystalline, the diorite clast-Sample 448-50-3, [80]-is included, because it represents a crystalline product of a more fractionated member of the arc-tholeiitic series.) 




Figure 13. Normative ternary feldspar diagram, showing the compositions of plagioclase from holocrystalline rocks. (Brackets show the degree of zoning of indicated units.)

Ridge can readily be summarized as being predominately an arc-tholeiitic series that is controlled principally by pyroxene-plagioclase fractionation. Local calc-alkalic volcanism may exist, but more conclusive evidence must be sought before this possibility is accepted.

Clearly more extensive studies of glasses and phases should be done to identify detailed mechanisms of fractionation. Careful correlations of the chemistry of the Palau-Kyushu Ridge with the West Mariana Ridge and the presently active Mariana Arc should also be done. For example, trace-element data do not even exist for the compositions of the active volcanoes in the Mariana Arc, it is not possible to determine whether they are calc-alkalic or not. Detailed petrochemical studies of exposed volcanic units on the arc must also be conducted to characterize the petrologic evolution of the frontal arc since $40 \mathrm{~m}$.y. ago; these results could be compared to results of studies of the remnant arcs to derive an integrated petrologic evolution of the island arc-back basin system.

\section{ACKNOWLEDGMENTS}

This paper is Texas A\&M University Geodynamics Research Program Contribution No. 10.

\section{REFERENCES}

Bass, M. N., Mobely, R., Rhodes, J. M., et al., 1973. Volcanic rocks cored in the central Pacific, Leg 17: Deep Sea Drilling Project. In Winterer, E. L., Ewing, J. I., et al., Init. repts. DSDP, 17: Washington (U.S. Govt. Printing Office), 429-446.
Carmichael, I. S. E., Turner, F. J., and Verhoogen, J., 1974. Igneous Petrology: New York (McGraw-Hill).

Fischer, A. G., Heezen, B. C., et al., 1971. Init. Rept. DSDP, 6: Washington (U.S. Govt. Printing Office).

Gill, J. B., 1970. Geochemistry of Viti Levu, Fiji and its evolution as an island arc. Contrib. Mineral. Petrol., 27:179-187. , 1976. Composition and age of Lua Basin and Ridge volcanic rocks: implications for evolution of an interarc basin and remnant arc. Bull. Geol. Soc. Am., 87:1384-1395.

Hajash, A., 1975. Hydrothermal processes along mid-ocean ridges : an experimental investigation. Contrib. Mineral. Petrol., 53: 205-226.

Hilde, T. W. C., Uyeda, S., and Kroenke, L., 1977. Evolution of the western Pacific and its margins. Tectonophysics, 30:145-165.

Ingle, J. C., Karig, D. E., et al., 1975. Site 296. In Karig, D. E., Ingle, J. C., Jr., et al., Init. Repts. DSDP, 31: Washington (U.S. Govt. Printing Office), 191-275.

International Working Group on the IGCP Project "Ophiolites," 1977. Initial report of the geological study of the ocean crust of the Philippine Sea floor. In Bogdanov, N. (Ed.), Bolletino del Grupo di Lavoro aulle Ofioliti Mediterranee, (Vol. 2): Bologna (Estratto da Ofioliti), 137-168.

Jakeš, P., and Gill, J., 1970. Rare-earth elements and the island-arc tholeiite series. Earth Planet. Sci. Lett., 9:17-28.

Johnson, J. R., 1979. Transitional basalts and tholeiites from the East Pacific Rise, $9^{\circ}$ N. J. Geophys. Res., 84:1635-1651.

Karig, D. E., 1971. Site surveys in the Mariana area (Scan IV). In Fischer, A. G., Heezen, B. C., et al., Init. Repts. DSDP, 6: Washington (U.S. Govt. Printing Office), 681-689.

, 1972. Remnant arcs. Bull. Geol. Soc. Am., 83:1057-1068.

1975a. Basin genesis in the Philippine Sea. In Karig, D. E., Ingle, J. C., et al., Init. Repts. DSDP, 31: Washington (U.S. Govt. Printing Office), 857-879.

1975b. Origin and development of marginal basins in the western Pacific. J. Geophys. Res., 76:2542-2560.

Karig, D. E., Ingle, J. C., Jr., et al., 1975. Init. Repts. DSDP, 31: Washington (U.S. Govt. Printing Office).

Kuno, H., 1965. Fractionation trends of basalt magmas in lava flows. J. Petrol., 6:302-321.

Miyashiro, A., 1974. Volcanic rock series in island arcs and active continental margins. Am. J. Sci., 274:321-355.

Scott, R. B., and Hajash, A., 1976. Initial submarine alteration of basaltic pillow lavas. Am. J. Sci., 276:480-501.

Shibata, T., 1976. Phenocryst-bulk rock composition relations of abyssal tholeiites and their petrogenic signficance. Geochim. Cosmochim. Acta, 40:1407-1417.

Shih, T. C., in press. Magnetic lineations in the Shikoku Basin. In Klein, G. deV., Kobayashi, K., et al., Init. Repts. DSDP, 58: Washington (U.S. Govt. Printing Office).

Taylor, S. R., 1969. Trace-element chemistry of andesite and associated calc-alkaline rocks. In McBirney, A. R. (Ed.), Proceedings of the Andesite Conference, Bulletin 65: Oregon (Dept. of Geology and Mineral Industries), pp. 43-63.

Tiezzi, L. J., and Scott, R. B., in press. Crystal fractionation in a cumulate gabbro, Mid-Atlantic Ridge, $26^{\circ} \mathrm{N}$. Lat. J. Geophys. Res.

Uyeda S., and Ben-Avraham, Z., 1972. Origin and development of the Philippine Sea. Nature Phys. Sci., 240:176-178.

Watanabe, T., Langseth, M. G., and Anderson, R. N., 1977. Heat flow in back-arc basin of the western Pacific. In Talwani, M., and Pitman, W. C., III (Ed.), Island Arcs, Deep-Sea Trenches, and Back-arc Basins: Washington (Am. Geophys. Union), pp. 137161.

Watts, A. G., and Weissel, J. K., 1975. Tectonic history of the Shikoku marginal basin. Earth Planet. Sci. Lett., 25:239-250. 